Network Working Group

Request for Comments: 3592

Obsoletes: 2558

Category: Standards Track
K. Tesink

Telcordia Technologies

September 2003

\title{
Definitions of Managed objects for the Synchronous Optical Network/Synchronous Digital \\ Hierarchy (SONET/SDH) Interface Type
}

Status of this Memo

This document specifies an Internet standards track protocol for the Internet community, and requests discussion and suggestions for improvements. Please refer to the current edition of the "Internet Official Protocol Standards" (STD 1) for the standardization state and status of this protocol. Distribution of this memo is unlimited.

Copyright Notice

Copyright (C) The Internet Society (2003). All Rights Reserved.

Abstract

This memo defines a portion of the Management Information Base (MIB) for use with network management protocols in TCP/IP-based internets. In particular, it defines objects for managing synchronous optical Network/Synchronous Digital Hierarchy (SONET/SDH) interfaces. This document is a companion to the documents that define Managed objects for the DS1/E1/DS2/E2 and DS3/E3 Interface Types.

This memo replaces RFC 2558. Changes relative to RFC 2558 are summarized in the MIB module's REVISION clause.

Table of Contents

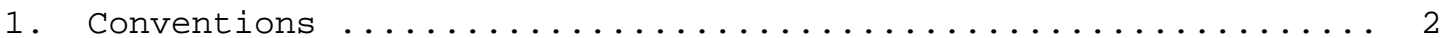

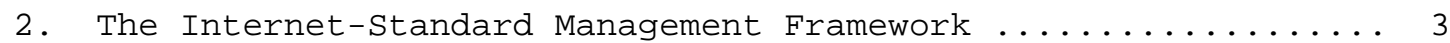

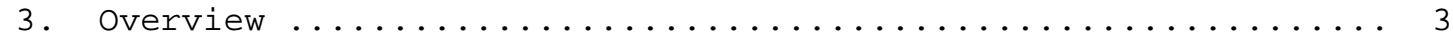

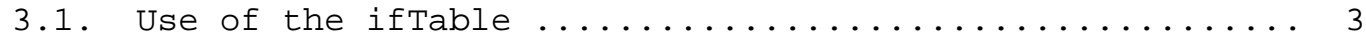

3.2. Use of ifTable for SONET/SDH

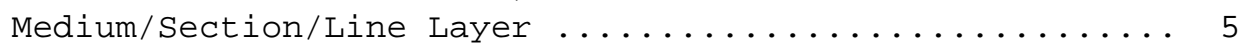

3.3. Use of iftable for SONET/SDH Paths ............... 6

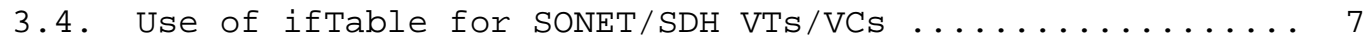

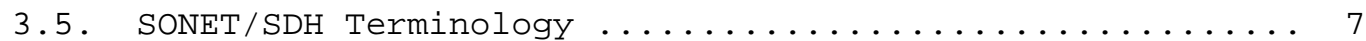

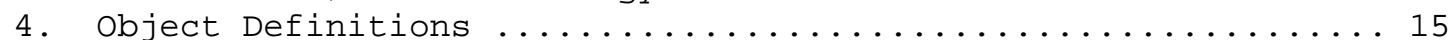

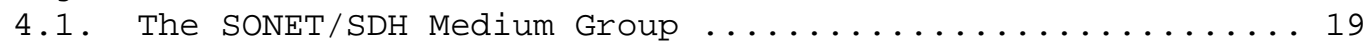

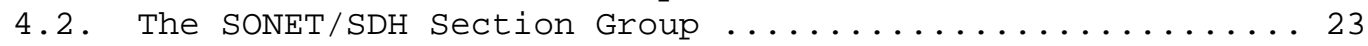


4.2.1. The SONET/SDH Section Current Group .......... 23

4.2.2. The SONET/SDH Section Interval Group ........ 25

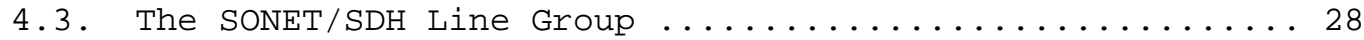

4.3.1. The SONET/SDH Line Current Group ............ 28

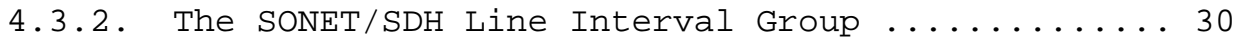

4.4. The SONET/SDH Far End Line Group ................. 32

4.4.1. The SONET/SDH Far End Line Current Group ...... 32

4.4.2. The SONET/SDH Far End Line Interval Group ..... 34

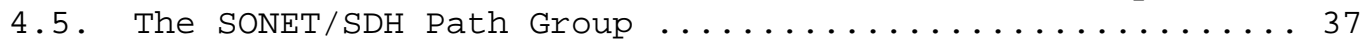

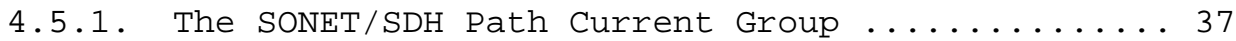

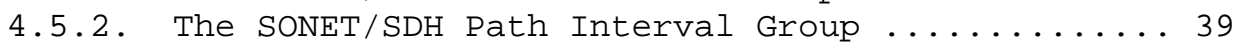

4.6. The SONET/SDH Far End Path Group ................. 42

4.6.1. The SONET/SDH Far End Path Current Group ...... 42

4.6.2. The SONET/SDH Far End Path Interval Group .....44 4

4.7. The SONET/SDH Virtual Tributary Group ............. 46

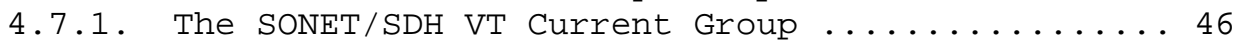

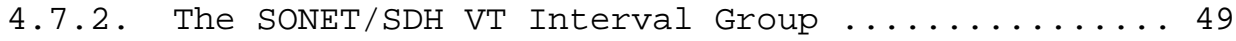

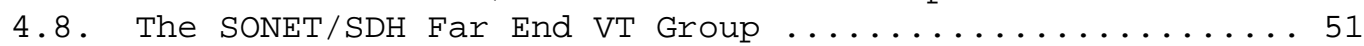

4.8.1. The SONET/SDH Far End VT Current Group ....... 51

4.8.2. The SONET/SDH Far End VT Interval Group....... 53

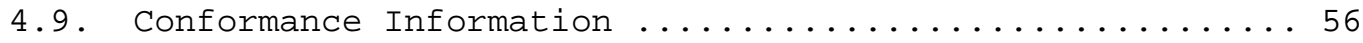

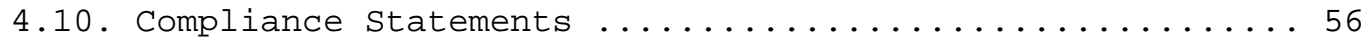

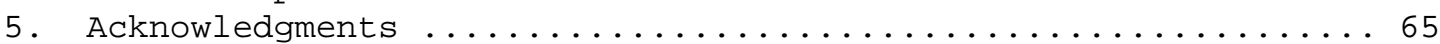

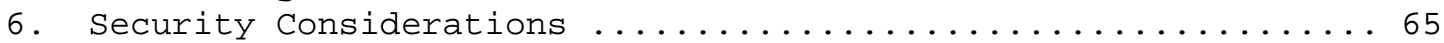

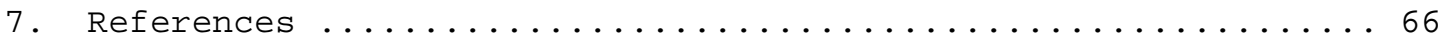

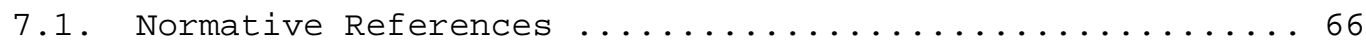

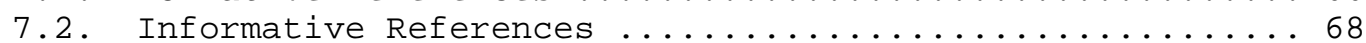

8. Intellectual Property statement ...................668

Appendix A: The delay-line approach to statistics collection .... 69

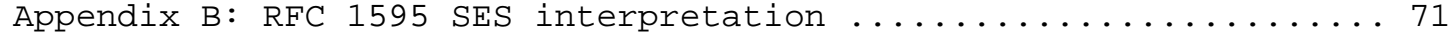

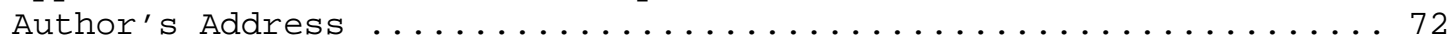

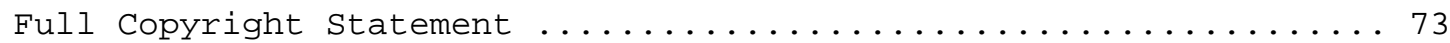

1. Conventions

The key words "MUST", "MUST NOT", "REQUIRED", "SHALL", "SHALL NOT", "SHOULD", "SHOULD NOT", "RECOMMENDED", "NOT RECOMMENDED", "MAY", and "OPTIONAL", when they appear in this document, are to be interpreted as described in BCP 14, RFC 2119 [RFC2119]. 
2. The Internet-Standard Management Framework

For a detailed overview of the documents that describe the current Internet-Standard Management Framework, please refer to section 7 of RFC 3410 [RFC3410].

Managed objects are accessed via a virtual information store, termed the Management Information Base or MIB. MIB objects are generally accessed through the Simple Network Management Protocol (SNMP). Objects in the MIB are defined using the mechanisms defined in the Structure of Management Information (SMI). This memo specifies a MIB module that is compliant to the SMIV2, which is described in STD 58, RFC 2578 [RFC2578], STD 58, RFC 2579 [RFC2579] and STD 58, RFC 2580 [RFC2580].

Textual conventions used in this document are defined in RFC 2579 [RFC2579] and RFC 3593 [RFC3593].

3. Overview

These objects are used when the particular media being used to realize an interface is a SONET/SDH interface. At present, this applies to these values of the iftype variable in the Internetstandard MIB:

sonet (39), sonetPath (50), sonetVT (51)

The definitions contained herein are based on the SONET/SDH specifications in ANSI T1.105 and T1.106-1988

[T1.105a][T1.105b] [T1.106] and CCITT G.707, 708, 709, and G.783 [G.707][G.708][G.709][G.783].

\subsection{Use of the ifTable}

This section specifies how the MIB II interfaces group, as defined in [RFC2863], is used for SONET/SDH interfaces. The SONET/SDH layers support several multiplexing possibilities.

For example in SONET, an Synchronous Transport Signal 3 (STS-3) has 3 SONET Paths, and a STS-3C has 1 SONET Path. Another example could be a STS-12 having 4 SONET STS-3c Paths. Similarly, a SONET Synchronous Payload Envelope (SPE) can carry many Virtual Tributaries (VTs), for example, one SONET SPE can carry $28 \mathrm{VT} 1.5 \mathrm{~s}$. It is important to note that an SPE and a VT in SONET is collectively referred to as a Virtual Container (VC) in SDH. Also, an STS is called Synchronous Transport Module (STM) in SDH. 
Not all SONET/SDH equipment terminates all SONET/SDH layers. For example, a SONET/SDH STE regenerator terminates SONET/SDH Sections only, and is transparent for all layers above that. SONET/SDH AddDrop multiplexers and Digital Cross Connect Systems terminate SONET/SDH Lines. SONET/SDH Terminal Multiplexers may also terminate SONET/SDH Paths and VTs/VCs.

MIB II [RFC1213], as extended by [RFC2863], accommodates these cases by appropriate use of the MIB II system group, and the interfaces group. The system group can name and describe the type of managed resource. The interfaces group defines which SONET/SDH layers apply, how these layers are configured and multiplexed. This is achieved by proper representation of SONET/SDH Layers by ifEntries as defined in [RFC2863], as follows:

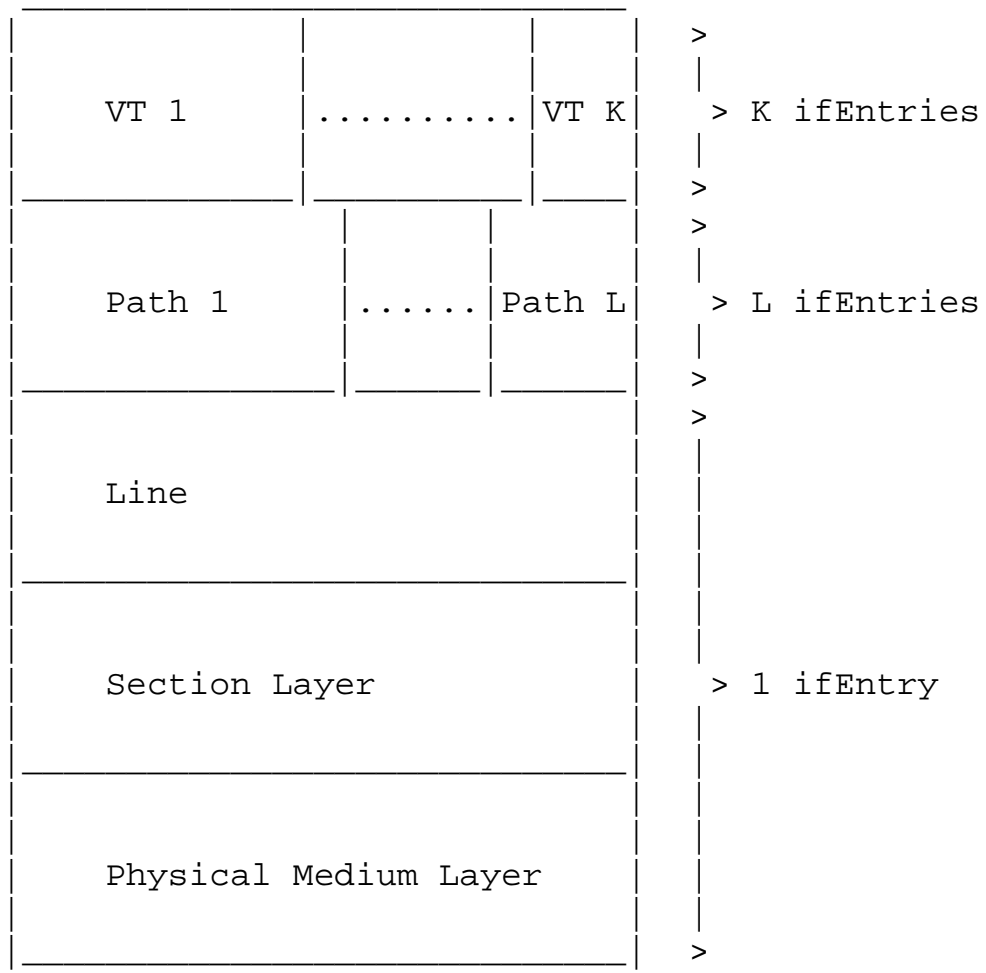

Use of iftable for a SONET/SDH port

The exact configuration and multiplexing of the layers is maintained in the ifstackTable [RFC2863] and in the ifInvStackTable [RFC2864]. 
3.2. Use of ifTable for SONET/SDH Medium/Section/Line Layer

Only the ifGeneralinformationGroup needs to be supported.

iftable Object Use for combined SONET/SDH

Medium/Section/Line Layer

\begin{tabular}{|c|c|}
\hline ifIndex & Interface index. \\
\hline ifDescr & SONET/SDH Medium/Section/Line \\
\hline iftype & sonet (39) \\
\hline ifspeed & $\begin{array}{l}\text { Speed of line rate for SONET/SDH, } \\
(\text { e.g., } 155520000 \text { bps). }\end{array}$ \\
\hline ifPhysAddress & $\begin{array}{l}\text { The value of the Circuit Identifier. } \\
\text { If no Circuit Identifier has been assigned } \\
\text { this object should have an octet string with } \\
\text { zero length. }\end{array}$ \\
\hline ifAdminstatus & $\begin{array}{l}\text { May be implemented with read-only access. } \\
\text { The desired administrative status of the } \\
\text { interface. }\end{array}$ \\
\hline ifoperstatus & $\begin{array}{l}\text { The value testing (3) is not used. } \\
\text { This object assumes the value down(2), } \\
\text { if the objects sonet SectionCurrentstatus } \\
\text { and sonetLineCurrentstatus have } \\
\text { any other value than sonetsectionNoDefect (1) } \\
\text { and sonetLineNoDefect (1), respectively. }\end{array}$ \\
\hline ifLastChange & sysupTime at the last change in ifoperstatus. \\
\hline ifName & $\begin{array}{l}\text { Textual name of the interface or an OCTET STRING } \\
\text { of zero length. }\end{array}$ \\
\hline \multicolumn{2}{|c|}{ ifLinkUpDownTrapEnable } \\
\hline ifHighspeed & $\begin{array}{l}\text { Speed of line in Mega-bits per second } \\
\text { (e.g., } 155 \text { Mbps) }\end{array}$ \\
\hline ifConnectorPresent & Set to true (1). \\
\hline ifAlias & $\begin{array}{l}\text { The (non-volatile) alias name for this interface } \\
\text { as assigned by the network manager. }\end{array}$ \\
\hline
\end{tabular}

Tesink

Standards Track

[Page 5] 


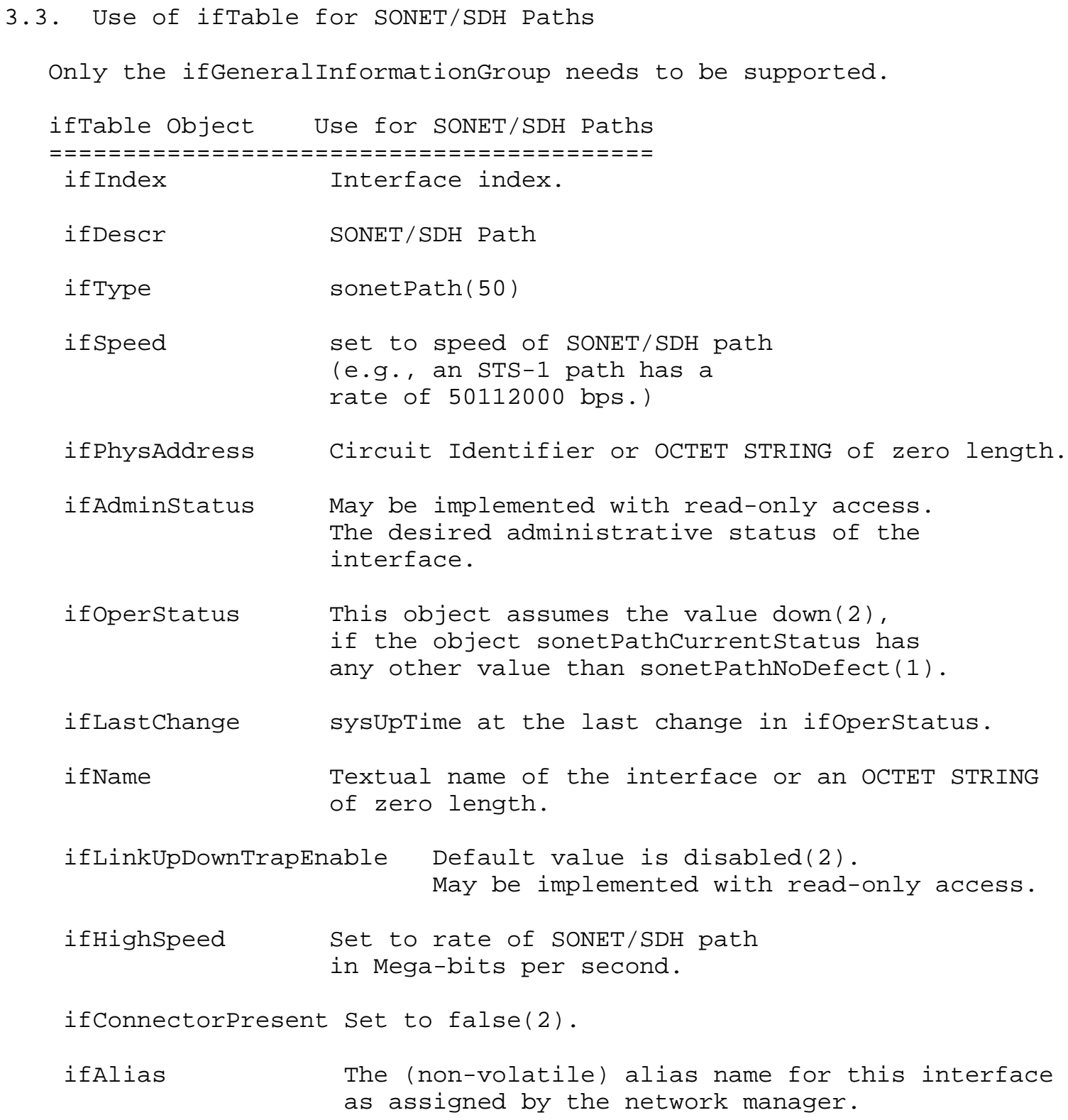


3.4. Use of ifTable for SONET/SDH VTs/VCs

Only the ifGeneralInformationGroup needs to be supported.

iftable Object Use for SONET/SDH VTs/VCs

$================================$

ifIndex

Interface index.

ifDescr SONET/SDH VT/VC

iftype sonetVT (51)

ifspeed Set to speed of VT/VC

(e.g., a VT1.5 has a rate of 1728000 bps.)

ifPhysAddress Circuit Identifier or OCTET STRING of zero length.

ifAdminstatus May be implemented with read-only access. The desired administrative status of the interface.

ifoperstatus This object assumes the value down(2), if the object sonetVTCurrentstatus has any other value than sonetVTNoDefect $(1)$.

iflastchange sysupTime at the last change in ifoperstatus.

ifName Textual name of the interface or an OCTET STRING of zero length.

ifLinkUpDownTrapEnable Default value is disabled(2).

May be implemented with read-only access.

ifHighspeed Set to rate of VT in Mega-bits per second.

ifConnectorPresent set to false(2).

ifAlias The (non-volatile) alias name for this interface as assigned by the network manager.

\subsection{SONET/SDH Terminology}

The terminology used in this document to describe error conditions on a SONET circuit as monitored by a SONET system are from the T1.231 [T1M1.3][T1.231a][T1.231b]. The terminology used in this document to describe error conditions on a SDH circuit as monitored by a SDH system are from the CCITT G.783 [G.783]. Only the SONET Performance 
Monitoring terminology is defined in this document. The definitions for SDH Performance Monitoring terms are similar but not identical, and they can be found in [G.783]. If the definition in this document does not match the definition in the T1.231 document, the implementer should follow the definition described in this document. In some cases other or additional references are used as compared with the ones cited above. This will be indicated in the text.

Section Loss of Frame Failure (Out of Frame Event, Severely

Errored Frame Defect) An Out of Frame (OOF) event (or Severely

Errored Frame defect) is the occurrence of four contiguous errored frame alignment words. A frame alignment word occupies the A1 and A2 bytes of an STS frame, and is defined in T1.105. The SEF defect is terminated when two contiguous error-free frame words are detected. Any implementation of the frame recovery circuitry which achieves realignment following an OOF within the 250 microsecond (two frames) interval implied by this definition is acceptable.

A Loss of Frame (LOF) defect is declared when an OOF/SEF defect persists for a period of 3 milliseconds. The LOF defect is terminated when the incoming signal remains continuously in-frame for a period of $1 \mathrm{~ms}$ to $3 \mathrm{~ms}$.

A LOF failure is declared when the LOF defect persists for a period of $2.5+/-0.5$ seconds, except when an LOS defect or failure is present. The LOF failure is cleared when the LOS failure is declared, or when the LOF defect is absent for $10+/-$ 0.5 seconds.

Loss of Signal

The Loss of Signal (LOS) defect is declared when no transitions are detected on the incoming signal (before descrambling). The LOS defect is detected upon observing 2.3 to 100 microseconds of no transitions. The LOS defect is cleared after a 125 microsecond interval (one frame) during which no LOS defect is detected.

The LOS failure is declared when the LOS defect persists for a period of $2.5+/-0.5$ seconds, or if LOS defect is present when the criteria for LOF failure declaration have been met. The LOS failure is cleared when the Los defect is absent for a period of 10 +/- 0.5 seconds. Declaration of LOS failure clears any existing LOF failure. Clearing the LOS failure allows immediate declaration of the LOF failure if conditions warrant. 
STS-Path Loss of Pointer

A Loss of Pointer (LOP) defect is declared when either a valid pointer is not detected in eight consecutive frames, or when eight consecutive frames are detected with the New Data Flag (NDF) set to "1001" without a valid concatenation indicator (see ANSI T1.105). A LOP defect is terminated when either a valid pointer with a normal NDF set to "0110", or a valid concatenation indicator is detected for three contiguous frames. Incoming STSPath AIS shall not result in the declaration of a LOP defect.

An STS-Path LOP failure is declared when the STS-Path LOP defect persists for a period of $2.5+/-0.5$ seconds. A STS-Path LOP failure is cleared when the STS-Path LOP defect is absent for 10 $+/-0.5$ seconds.

VT Loss of Pointer

A VT LOP defect is declared when either a valid pointer is not detected in eight consecutive VT superframes, or when eight consecutive VT superframes are detected with the NDF set to "1001" without a valid concatenation indicator. A VT LOP defect is terminated when either a valid pointer with a normal NDF set to "0110", or a valid concatenation indicator is detected for three contiguous VT superframes. Incoming VT-Path AIS shall not result in declaring a VT LOP defect.

A VT LOP failure is declared when the VT LOP defect persists for $2.5+/-0.5$ seconds. A VT LOP failure is cleared when the VT LOP defect is absent for $10+/-0.5$ seconds.

Line Alarm Indication Signal

A Line Alarm Indication Signal (L-AIS) is defined in ANSI T1.105. The following criteria are specific to the L-AIS defect:

- Line AIS defect is detected as a "111" pattern in bits 6, 7, and 8 of the $\mathrm{K} 2$ byte in five consecutive frames.

- Line AIS defect is terminated when bits 6, 7, and 8 of the K2 byte do not contain the code "111" for five consecutive frames.

A Line AIS failure is declared when the Line AIS defect persists for a period of $2.5+/-0.5$ seconds. A Line AIS failure is cleared when the Line AIS defect is absent for $10+/-0.5$ seconds.

STS-Path Alarm Indication Signal

The STS-Path Alarm Indication Signal (AIS) is defined in ANSI $\mathrm{T} 1.105$ as all ones in bytes $\mathrm{H} 1, \mathrm{H} 2$, and $\mathrm{H} 3$ as well as all ones in the entire STS SPE. The following criteria are specific to the STS-Path AIS defect: 
- STS-Path AIS defect is detected as all ones in bytes H1 and H2 in three contiguous frames.

- The STS-Path AIS defect is terminated when a valid STS Pointer is detected with the NDF set to "1001" (inverted) for one frame, or "0110" (normal) for three contiguous frames.

An STS-Path AIS failure is declared when the STS-Path AIS defect persists for $2.5+/-0.5$ seconds. An STS-Path AIS failure is cleared when the STS-Path AIS defect is absent for $10+/-0.5$ seconds.

VT-Path Alarm Indication Signal

The VT-Path Alarm Indication Signal (AIS) is only applicable for VTs in the floating mode of operation. VT-Path AIS is used to alert the downstream VT Path Terminating Entity (PTE) of an upstream failure. Upon detection of a failure, Line AIS, or STSPath AIS, an STS PTE will generate downstream VT-Path AIS if the STS Synchronous Payload Envelope (SPE) is carrying floating VTs. VT-Path AIS is specified in ANSI T1.105 as all ones in bytes V1, V2, V3, and V4, as well as all ones in the entire VT SPE. The following criteria are specific to VT-Path AIS defect:

- VT-Path AIS defect is detected by a VT PTE as all ones in bytes V1 and V2 in three contiguous VT superframes.

- VT-Path AIS defect is terminated when valid VT pointer with a valid VT size is detected with the NDF set to "1001" (inverted) for one VT superframe, or "0110" (normal) for three contiguous VT superframes are detected.

A VT-Path AIS failure is declared when the VT-Path AIS defect persists for $2.5+/-0.5$ seconds. A VT-Path AIS failure is cleared when the VT-Path AIS defect is absent for $10+/-0.5$ seconds.

Line Remote Defect Indication Line Remote Defect Indication (RDI) (aka Line FERF) signal is the occurrence of a "110" pattern in bit positions 6, 7, and 8 of the K2 byte in STS-1 \#1 of the STS-N signal. Line RDI is defined in ANSI T1.105. The following criteria are specific to Line RDI defect:

- Line RDI defect is a "110" code in bits 6, 7, and 8 of the K2 byte of in STS-1 \#1 in $x$ consecutive frames, where $x=5$ [T1.231a] [T1.231b] or 10 [T1.231b]. 
- Line RDI defect is terminated when any code other than "110" is detected in bits 6,7 , and 8 of the $\mathrm{K} 2$ byte in $x$ consecutive frames, where $\mathrm{x}=5$ [T1.231a][T1.231b] or 10 [T1.231b].

A Line Remote Failure Indication (RFI) failure is declared when the incoming Line RDI defects lasts for $2.5+/-0.5$ seconds. The Line RFI failure is cleared when no Line RDI defects are detected for $10+/-0.5$ seconds.

STS-Path Remote Defect Indication

STS-Path RDI (aka STS-Path FERF) signal shall be generated within 100 milliseconds by the STS PTE upon detection of an AIS or LOP defect. Transmission of the STS-Path RDI signal shall cease within 100 milliseconds when the STS PTE no longer detects STSPath AIS or STS-Path LOP defect. The STS-Path RDI shall accurately report the presence or absence of STS-Path AIS or STSPath LOP defects. STS-Path RDI defect is defined in ANSI T1.105. The following requirements are specific to the STS-Path RDI defect:

- STS-Path RDI is detected by all STS PTEs. STS-Path RDI is detected by the upstream STS PTE as a "1" in bit five of the Path status byte (G1) for $x$ consecutive frames, where $\mathrm{x}=5$ [T1.231a] or 10 [T1.231b].

- Removal of STS-Path Remote Defect Indication is detected by a "0" in bit 5 of the G1 byte in $\mathrm{x}$ consecutive frames, where $\mathrm{x}=$ 5 [T1.231a] or 10 [T1.231b].

An STS-Path Remote Failure Indication (RFI) failure is declared when the incoming STS-Path RDI defects lasts for $2.5+/-0.5$ seconds. The STS-Path RFI failure is cleared when no STS-Path RDI defects are detected for $10+/-0.5$ seconds.

VT-Path Remote Defect Indication

VT Path RDI (aka VT Path FERF) signal shall be generated within 100 milliseconds by the VT PTE upon detection of a VT-Path AIS or LOP defect. Transmission of the VT-Path RDI signal shall cease within 100 milliseconds when the VT PTE no longer detects VT-Path AIS or VT-Path LOP defect. The VT-Path RDI shall accurately report the presence or absence of VT-Path AIS or VT-Path LOP defects. VTPath RDI defect is defined in ANSI T1.105. The following requirements are specific to VT-Path RDI defect:

- VT-Path RDI defect is the occurrence of a "1" in bit 4 of the VT-Path Overhead byte (V5) in $\mathrm{x}$ consecutive frames, where $\mathrm{x}=5$ [T1.231a] or 10 [T1.231b]. 
- VT-Path RDI defect is terminated when a "0" is detected in bit 4 of the VT-Path Overhead byte (V5) for $x$ consecutive frames, where $\mathrm{x}=5$ [T1.231a] or 10 [T1.231b].

A VT-Path Remote Failure Indication (RFI) (derived) failure is declared when the incoming VT-Path RDI defects lasts for $2.5+/-$ 0.5 seconds. The VT-Path RFI failure is cleared when no VT-Path RDI defects are detected for $10+/-0.5$ seconds.

VT-Path Remote Failure Indication

The VT-Path RFI signal is only required for the case of byte synch mapped DS1s where the DS1 frame bit is not mapped. The VT-Path RFI is specified in ANSI T1.105, where it is currently called VT path yellow. When provided, the VT-Path RFI signal is used to indicate the occurrence of far-end failures. When the VT-Path RFI is not provided, far-end failures are derived from local timing of the VT-Path RDI defect. The VT-Path RFI failure is declared within $5 \mathrm{~ms}$ of detecting the incoming VT-Path RFI Signal. The VT-Path Remote Failure Indication (RFI) failure is cleared within $50 \mathrm{~ms}$ of detecting the removal of the incoming VT-Path RFI signal.

Coding Violation

Coding Violations (CV) are Bit Interleaved Parity (BIP) errors that are detected in the incoming signal. CV counters are incremented for each BIP error detected. That is, each BIP-8 can detect up to eight errors per STS-N frame, with each error incrementing the CV counter. Section CVs shall be collected using the BIP-8 in the B1 byte located in the section Overhead of STS-1 \#1. Line CVs shall be collected using the BIP-8s in B2 bytes located in the Line Overhead of each STS-1 (since all CVs on an STS-N line are counted together, this is equivalent to counting each error in the $B I P-8 * N$ contained in the B2 bytes of the STS-N Line Overhead). Thus, on an STS-N signal, up to 8 x $N$ CVs may occur in each frame. Path CVs shall be collected using the BIP-8 in the B3 byte of the STS-Path Overhead of the STS SPE. VT CVs shall be collected using the BIP-2 in the V5 overhead byte of the floating VT.

\section{Errored Seconds}

At each layer, an Errored Second (ES) is a second with one or more Coding Violations at that layer OR one or more incoming defects (e.g., SEF, LOS, AIS, LOP) at that layer has occurred.

Severely Errored Seconds

According to [T1M1.3][T1.231a][TR253][GR253][T1.231b] at each layer, an Severely Errored Second (SES) is a second with $x$ or more CVs at that layer, or a second during which at least one or more incoming defects at that layer has occurred. The values of $x$ in 
RFC 1595 [RFC1595] were based on [T1M1.3] and [TR253] (see Appendix B). These values have subsequently been relaxed in [T1.231a][GR253][T1.231b]. In addition, according to G.826 [G.826] SESs are measured as a percentage of errored blocks.

To deal with these sets of definitions this memo defines an object sonetSESthresholdSet that determines the correct interpretation of SES. For backward compatibility, if this object is not implemented the interpretation of Appendix B shall apply. Otherwise, a more recent interpretation is suggested. An agent is not required to support all sets of definitions.

Note that CV counts should be frozen during SESs.

Note that if a manager changes the value of this object all SES statistics collected prior to this change shall be invalidated.

Severely Errored Framing Seconds

A Severely Errored Framing Second (SEFS) is a second containing one or more SEF events. This counter is only counted at the Section Layer.

Unavailable Seconds

At the Line, Path, and VT layers, an unavailable second is calculated by counting the number of seconds that the interface is unavailable. At each layer, the SONET/SDH interface is said to be unavailable at the onset of 10 contiguous SESs. The 10 SESs are included in unavailable time. Once unavailable, the SONET/SDH interface becomes available at the onset of 10 contiguous seconds with no SESS. The 10 seconds with no SESs are excluded from unavailable time. With respect to the SONET/SDH error counts at each layer, all counters at that layer are incremented while the SONET/SDH interface is deemed available at that layer. While the interface is deemed unavailable at that layer, the only count that is incremented is UASs at that layer.

Note that this definition implies that the agent cannot determine until after a ten second interval has passed whether a given onesecond interval belongs to available or unavailable time. If the agent chooses to update the various performance statistics in real time then it must be prepared to retroactively reduce the ES, SES, and SEFS counts by 10 and increase the UAS count by 10 when it determines that available time has been entered. It must also be prepared to reduce the $\mathrm{CV}$ count by the number of violations counted since the onset of unavailable time. The agent must be similarly prepared to retroactively decrease the UAS count by 10 and increase the ES and CV counts as necessary upon entering available time. A special case exists when the 10 second period 
leading to available or unavailable time crosses a 900 second statistics window boundary, as the foregoing description implies that the CV, ES, SES, SEFS, and UAS counts the PREVIOUS interval must be adjusted. In this case successive GETs of the affected sonetPathIntervalSES and sonetPathIntervalUAS objects (and the analogous Line and VT objects also) objects will return differing values if the first GET occurs during the first few seconds of the window.

According to ANSI T1.231 unavailable time begins at the _onset_of 10 contiguous severely errored seconds -- that is, unavailable time starts with the ffirst_of the 10 contiguous SESs. Also, while an interface is deemed unavailable all counters for that interface are frozen except for the UAS count. It follows that an implementation which strictly complies with this standard must _not_increment any counters other than the UAS count -- even temporarily -- as a result of anything that happens during those 10 seconds. Since changes in the signal state lag the data to which they apply by 10 seconds, an ANSI-compliant implementation must pass the one-second statistics through a 10-second delay line prior to updating any counters. That can be done by performing the following steps at the end of each one second interval.

i) Read near/far end CV counter and alarm status flags from the hardware.

ii) Accumulate the CV counts for the preceding second and compare them to the ES and SES threshold for the layer in question. Update the signal state and shift the one-second CV counts and ES/SES flags into the 10-element delay line. Note that far-end one-second statistics are to be flagged as "absent" during any second in which there is an incoming defect at the layer in question or at any lower layer.

iii) Update the current interval statistics using the signal state from the _previous_ update cycle and the one-second CV counts and ES/SES flags shifted out of the 10-element delay line.

This approach is further described in Appendix A. An agent may choose to use this approach in lieu of retroactive adjustments to the counters.

In any case, a linkDown trap shall be sent only after the agent has determined for certain that the unavailable state has been entered, but the time on the trap will be that of the first UAS (i.e., 10 seconds earlier). A linkUp trap shall be handled similarly. 
Unequipped

If a Path or VT connection is not provisioned (idle) the SONET equipment will signal this state by transmitting the Path or VT Signal Label as follows: - byte C2 of the STS Path Overhead equal to 0 for an unequipped Path, - byte V5 of the VT Path Overhead equal to 0 for an unequipped VT.

Signal Label Mismatch

A Path or VT connection is not correctly provisioned if a received Path or VT Signal Label mismatch occurs. A received Signal Label is considered mismatched if it does not equal either the locally provisioned value or the value 'equipped non-specific' (1 hex). Note that any received non-zero Signal Label is considered a locally provisioned value of 'equipped non-specific'. Only inservice, provisioned Path Terminating equipment can detect mismatched Signal labels. It is considered provisioned if it has been configured for a mapping and has been assigned signals to and from which the mapping takes place. While a Path is unequipped or has mismatched signal labels ES/SES counts continue, but these conditions do not themselves contribute to ES/SES.

Circuit Identifier

This is a character string specified by the circuit vendor, and is useful when communicating with the vendor during the troubleshooting process.

4. Object Definitions

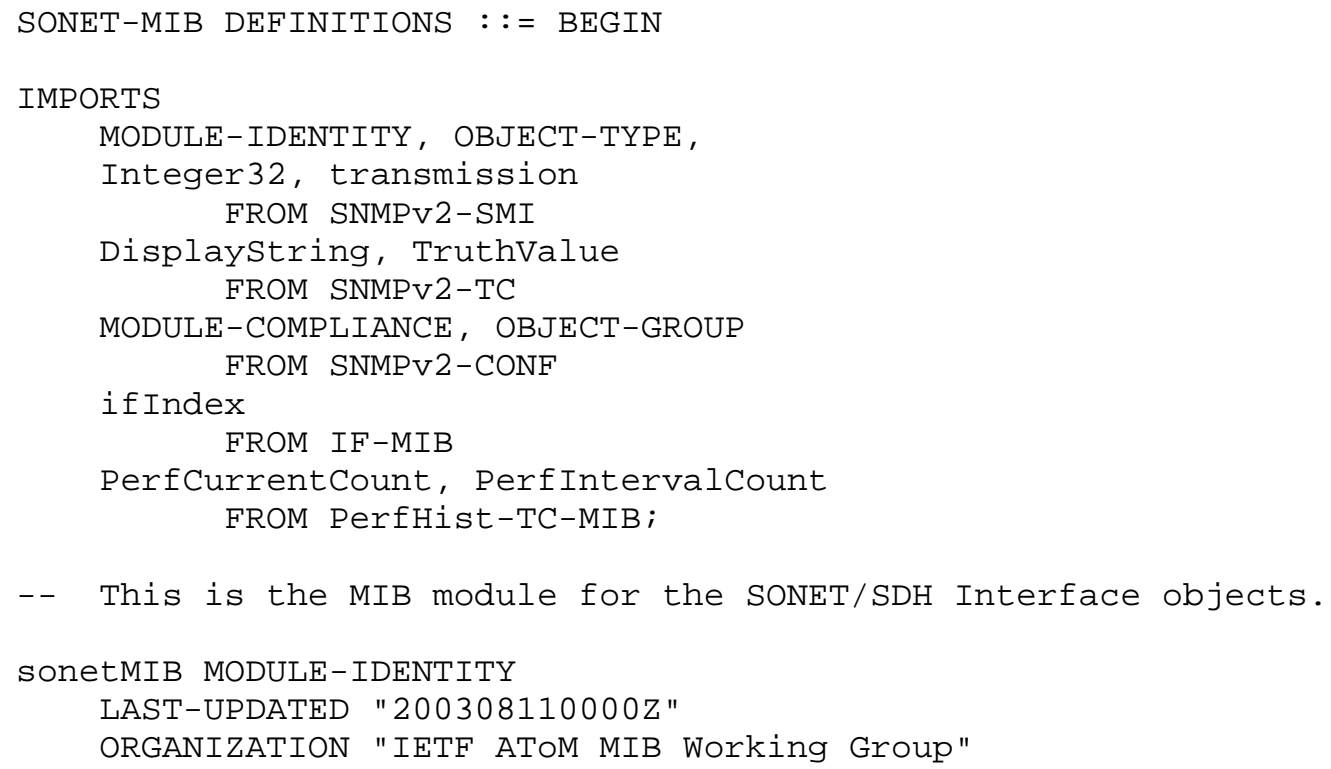




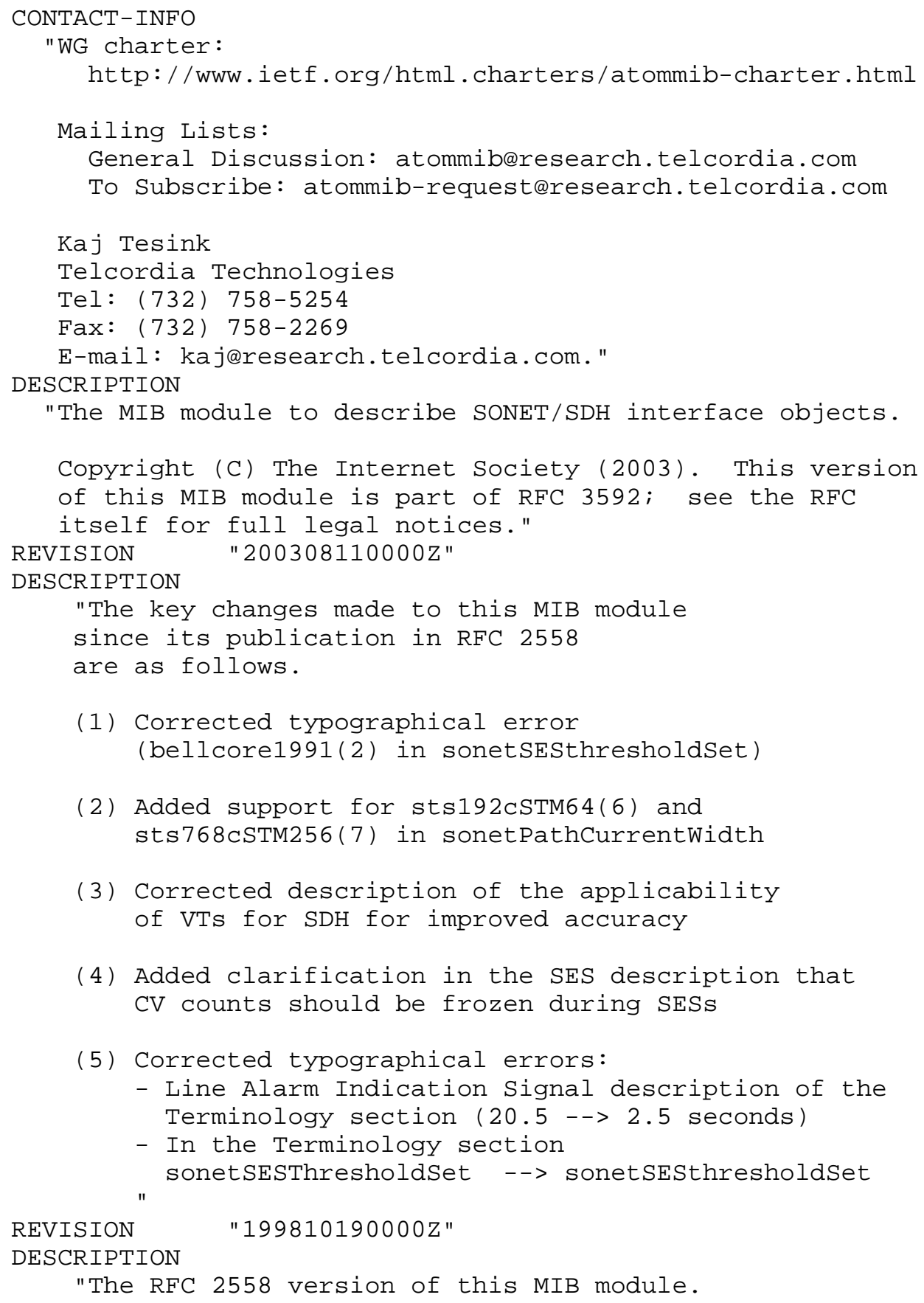

"The RFC 2558 version of this MIB module. 
The key changes made to this MIB module

since its initial publication in RFC 1595

are as follows.

(1) The MODULE-IDENTITY has been updated to reflect the changes to the MIB.

(2) Where applicable, the textual conventions PerfCurrentCount and PerfIntervalcount from PerfHist-TC-MIB have been used in place of Gauge 32 .

(3) An agent now has the option to delay updates to the various performance counts in lieu of performing retroactive adjustments upon entering into or exiting from unavailable time. This implementation option is described in Appendix A of this memo.

(4) In order to make the SONET-MIB more useful for circuit provisioning, the formerly read-only objects sonetMediumType, sonetMediumLineCoding, sonetMediumLineType, and sonetMediumCircuitIdentifier have been given a MAX-ACCESS of read-write. The MIN-ACCESS remains read-only.

(5) The DESCRIPTION clause for sonetMediumTimeElapsed has been updated to describe its behaviour if the duration of the current interval exceeds the maximum value.

(6) The DESCRIPTION clause for sonetMediumValidintervals has been updated to describe its behaviour when some intervals may be unavailable, and the object sonetMediumInvalidIntervals has been added to keep count of the number of missing intervals (if any).

(7) The object sonetMediumLoopbackConfig has been added to enable or disable loopback configurations.

(8) Because the error count thresholds for declaring severely errored seconds that are specified in ANSI T1.231-1993, ITU-T G.826-1995, and ANSI T1.231-1997 are all different from each other and from the thresholds specified in RFC 1595, an enumerated INTEGER object sonetSESthresholdSet has been added to allow an agent to specify which threshold set is in use. Text has been added to section 3 stating that if this object is not implemented the thresholds specified in RFC 1595 should be assumed, and the table containing those thresholds has been moved to Appendix B of this memo. 
(9) A column with SYNTAX TruthValue has been added to each interval table. The purpose of the additional column is to indicate, for each interval, whether the data is valid in the sense intended by ANSI T1.231 clause 9.1.2.2 [T1.231a][T1.231b]. The objects in question are:

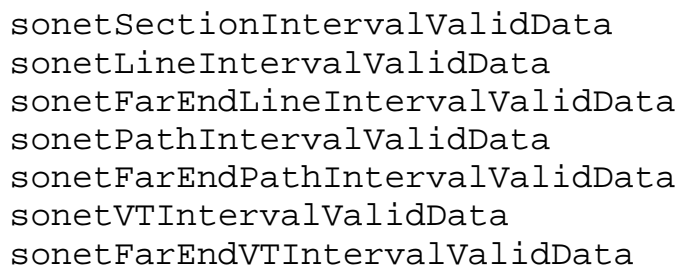

(10) The ranges for sonetPathcurrentstatus and sonetVTCurrentstatus have been made consistent with the DESCRIPTION clauses.

(11) The conformance information has been updated. Previous conformance information from RFC 1595 has been deprecated. Some typographical errors in the deprecated section have been corrected in order to prevent MIB compilation errors."

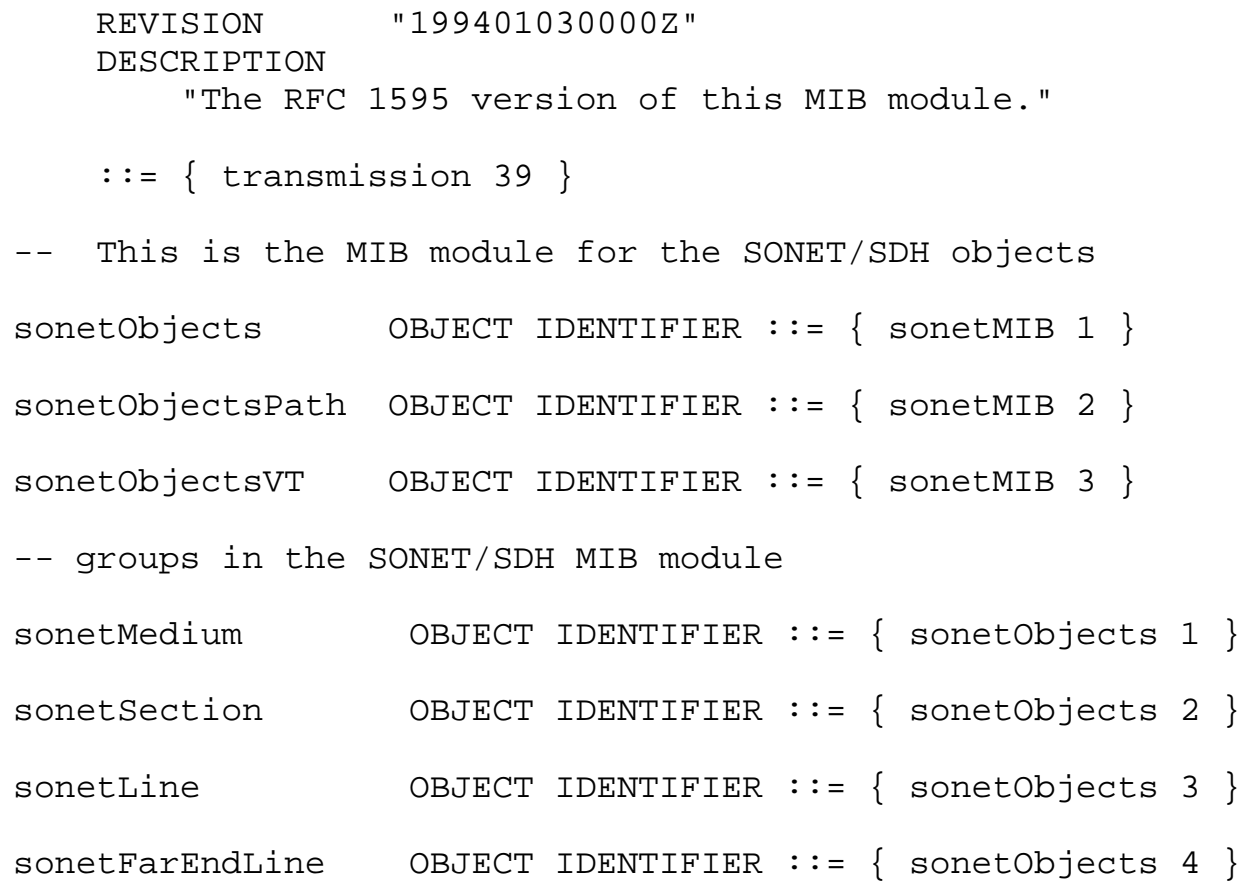




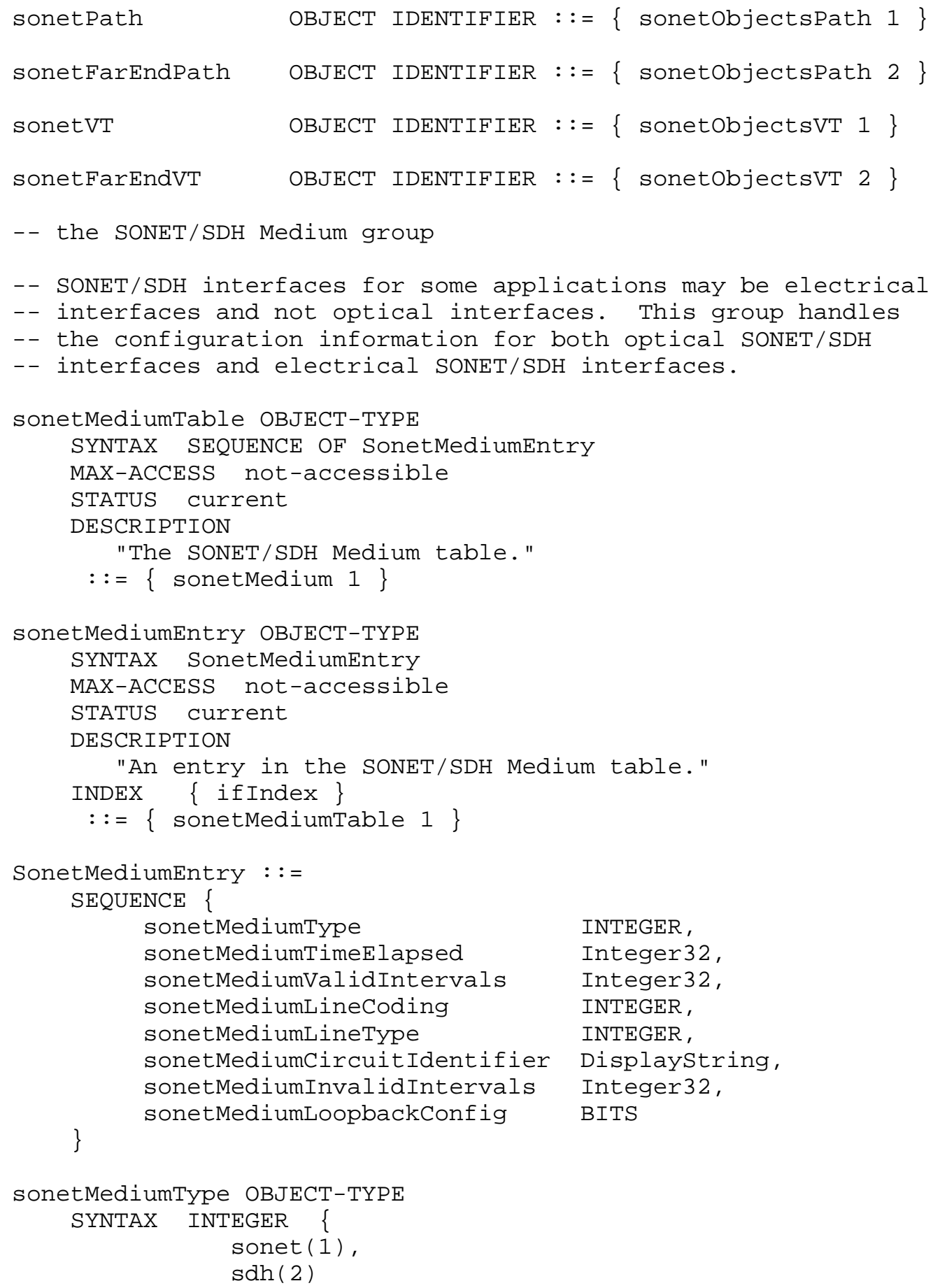




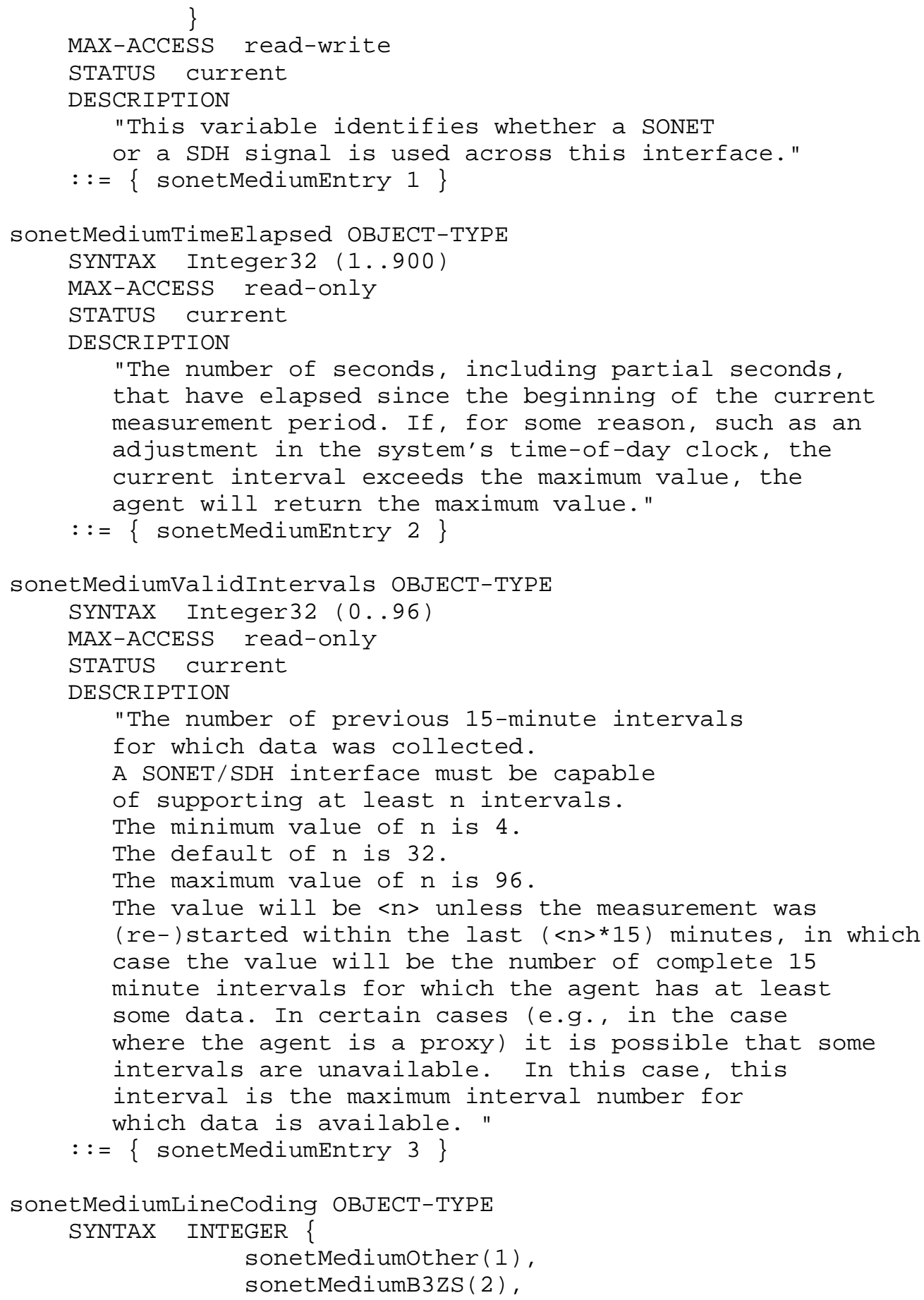




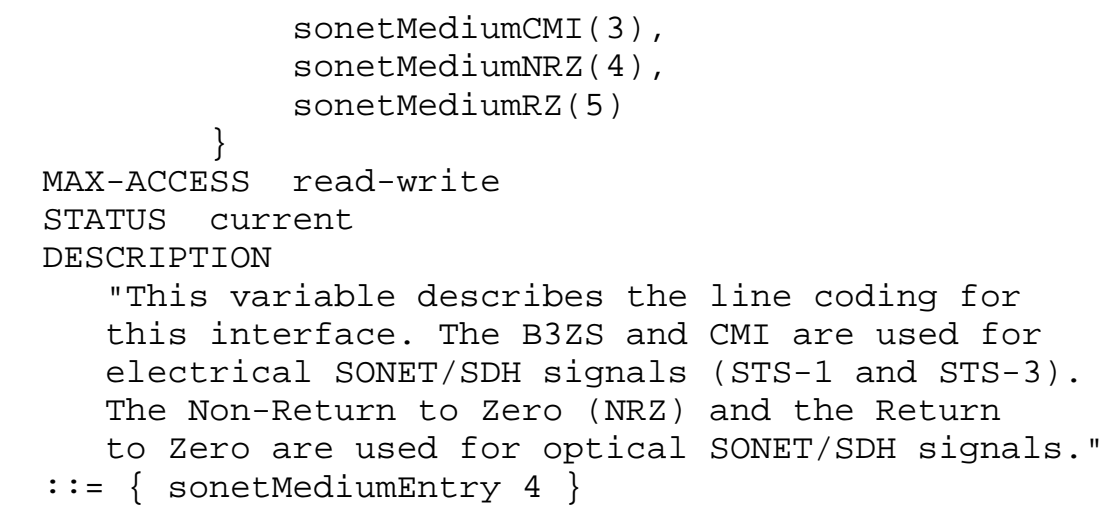




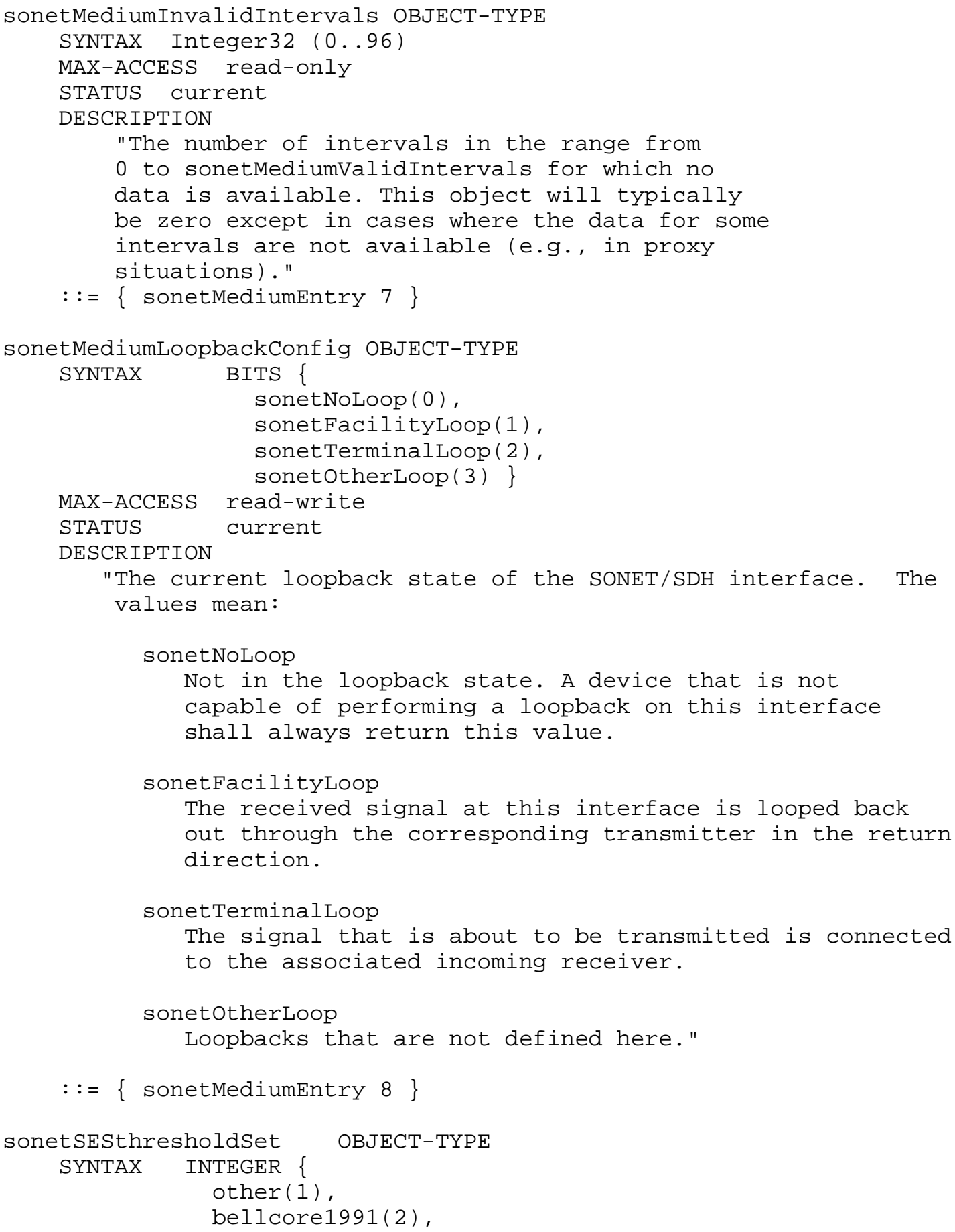




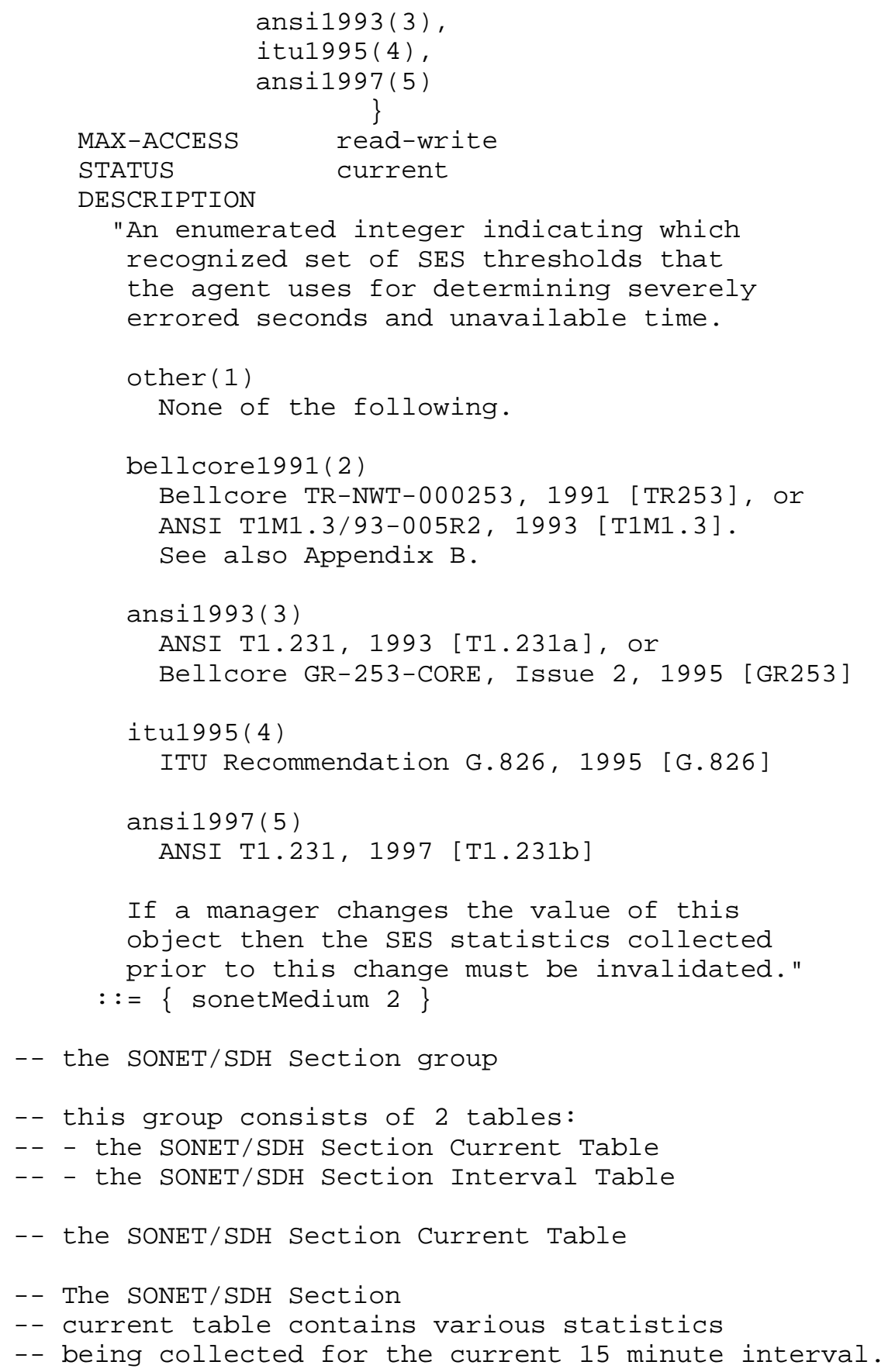




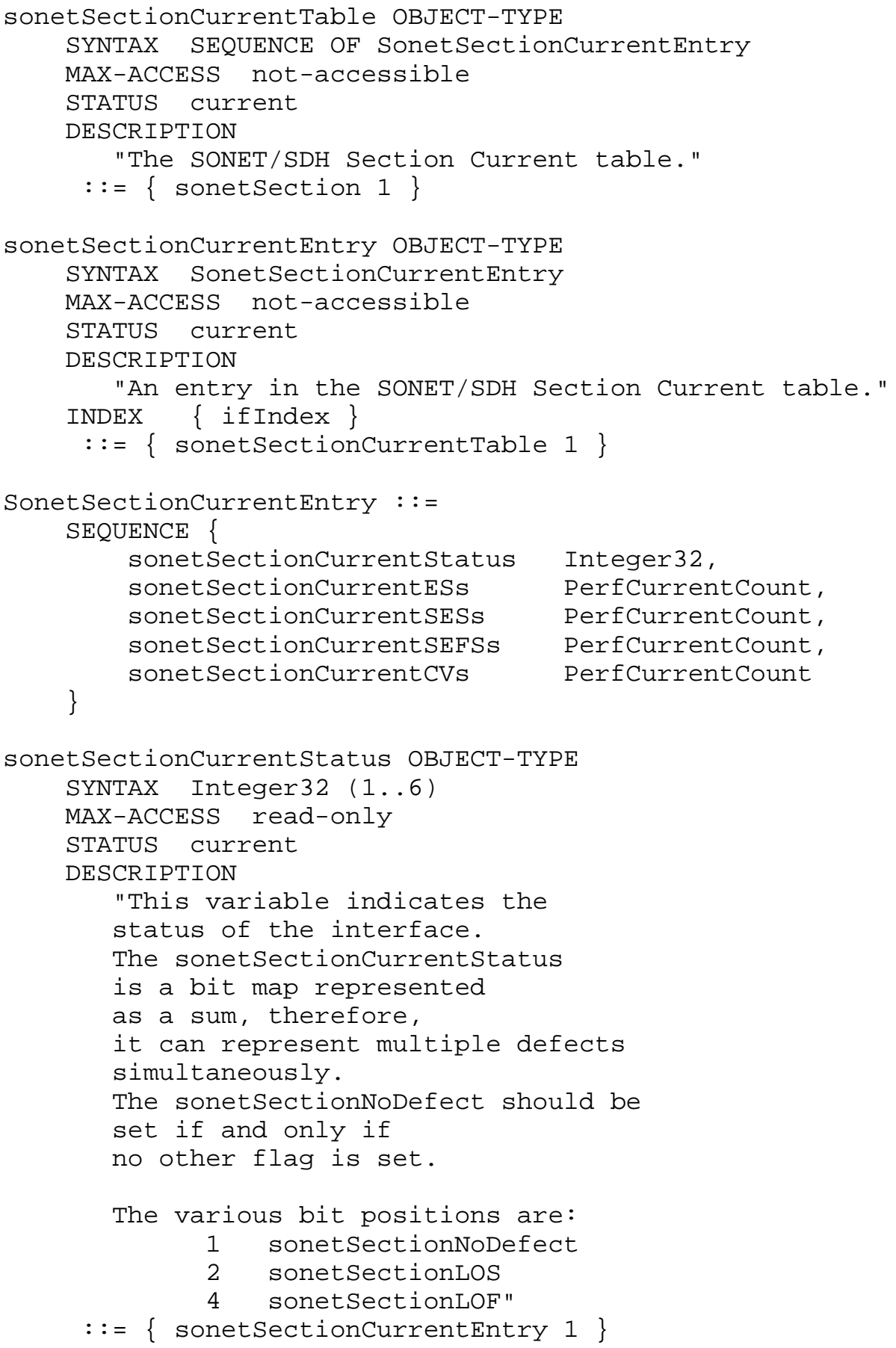




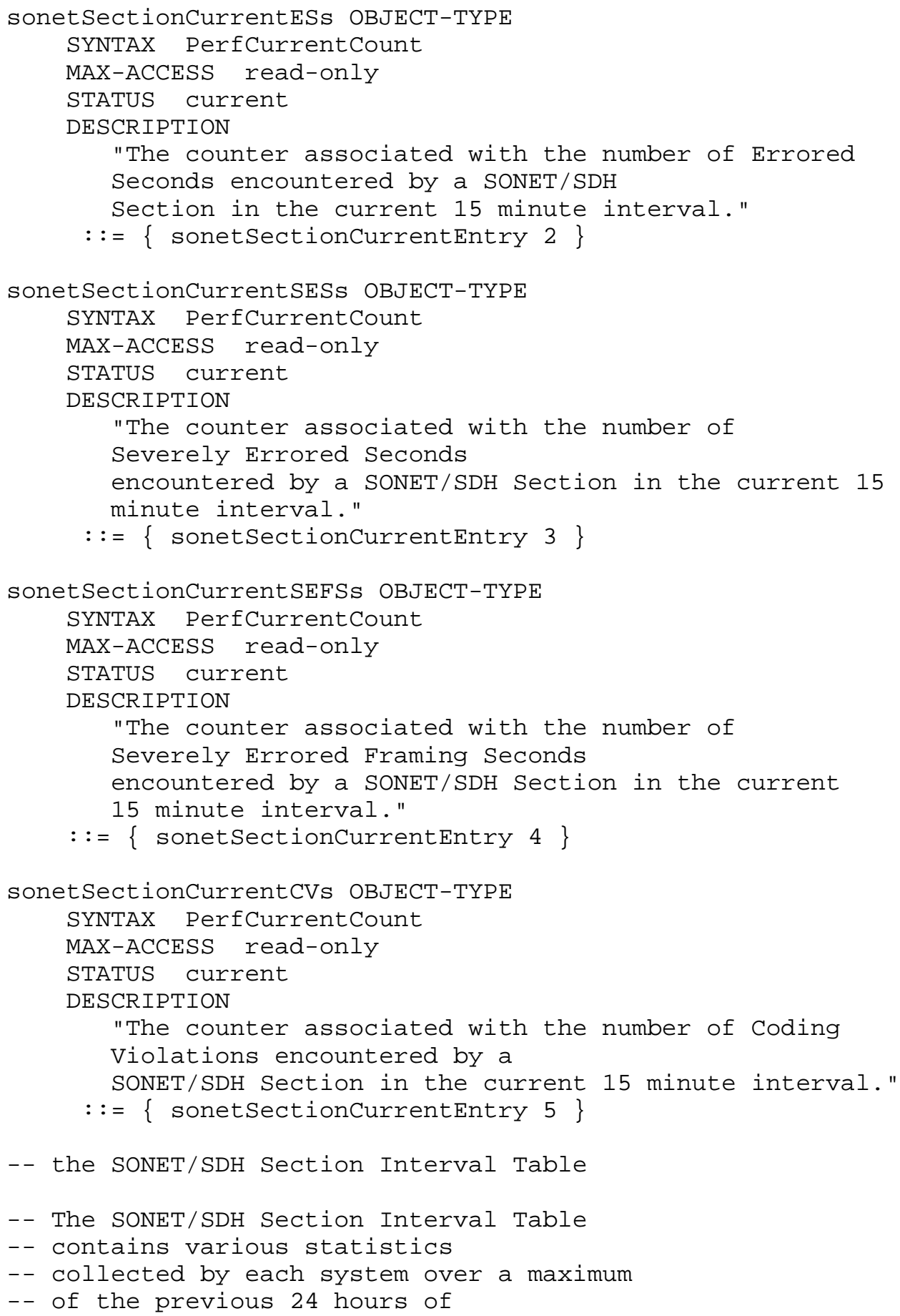


-- operation. The past 24 hours may be broken into 96

-- completed 15 minute intervals.

-- A system is required to store at

-- least 4 completed 15 minute interval.

-- The default value is 32 intervals.

sonet SectionIntervalTable OBJECT-TYPE

SYNTAX SEQUENCE OF SonetSectionIntervalEntry

MAX-ACCESS not-accessible

STATUS current

DESCRIPTION

"The SONET/SDH Section Interval table."

$::=\{$ sonetsection 2$\}$

sonetSectionIntervalEntry OBJECT-TYPE

SYNTAX SonetsectionIntervalEntry

MAX-ACCESS not-accessible

STATUS current

DESCRIPTION

"An entry in the SONET/SDH Section Interval table."

INDEX $\{$ ifIndex, sonetSectionIntervalNumber \}

$::=\{$ sonetSectionIntervalTable 1 \}

Sonet SectionIntervalEntry : :=

SEQUENCE \{

sonetSectionIntervalNumber Integer32, sonetsectionIntervaless PerfIntervalcount, sonetSectionIntervalSESs PerfIntervalcount, sonetSectionIntervalSEFSs PerfIntervalcount, sonetSectionIntervalcVs PerfIntervalcount, sonetSectionIntervalValidData TruthValue

sonet SectionIntervalNumber OBJECT-TYPE

SYNTAX Integer32 (1..96)

MAX-ACCESS not-accessible

STATUS current

DESCRIPTION

"A number between 1 and 96, which identifies the interval for which the set of statistics is available.

The interval identified by 1 is the most recently completed 15 minute interval,

and the interval identified

by $\mathrm{N}$ is the interval immediately preceding the

one identified

by $\mathrm{N}-1 . "$

$::=\{$ sonetSectionIntervalEntry 1$\}$

Tesink

Standards Track

[Page 26] 


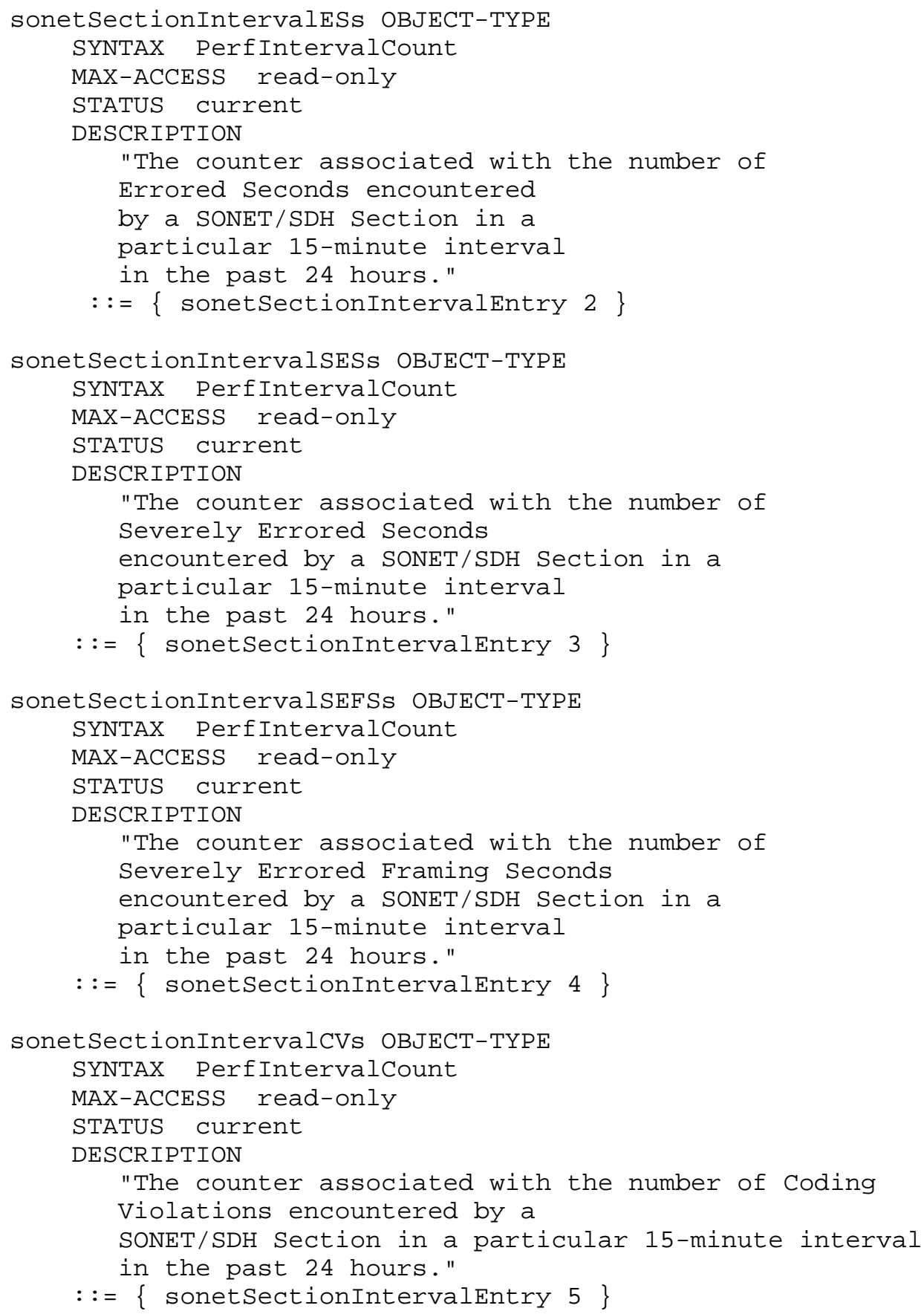




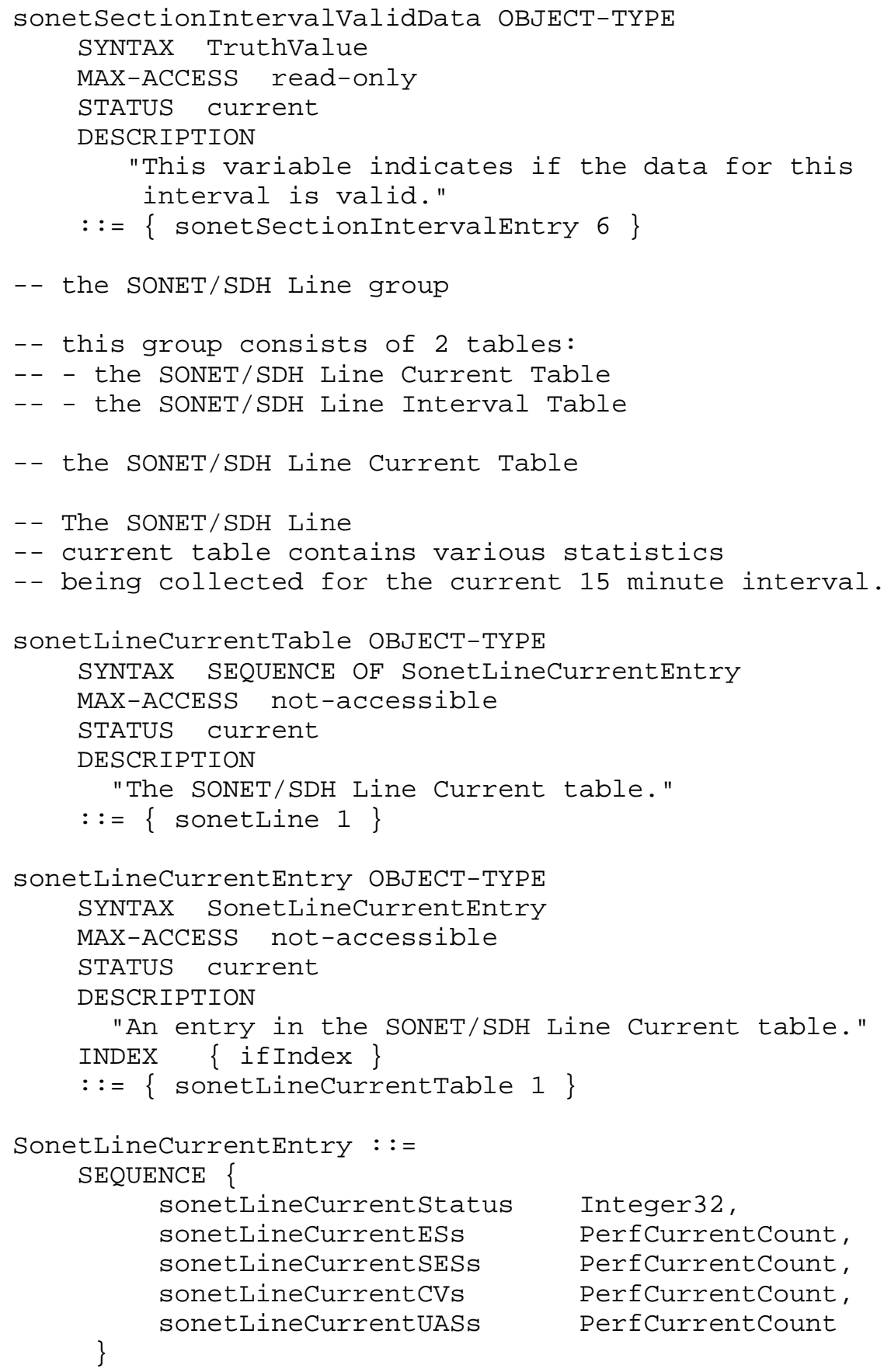




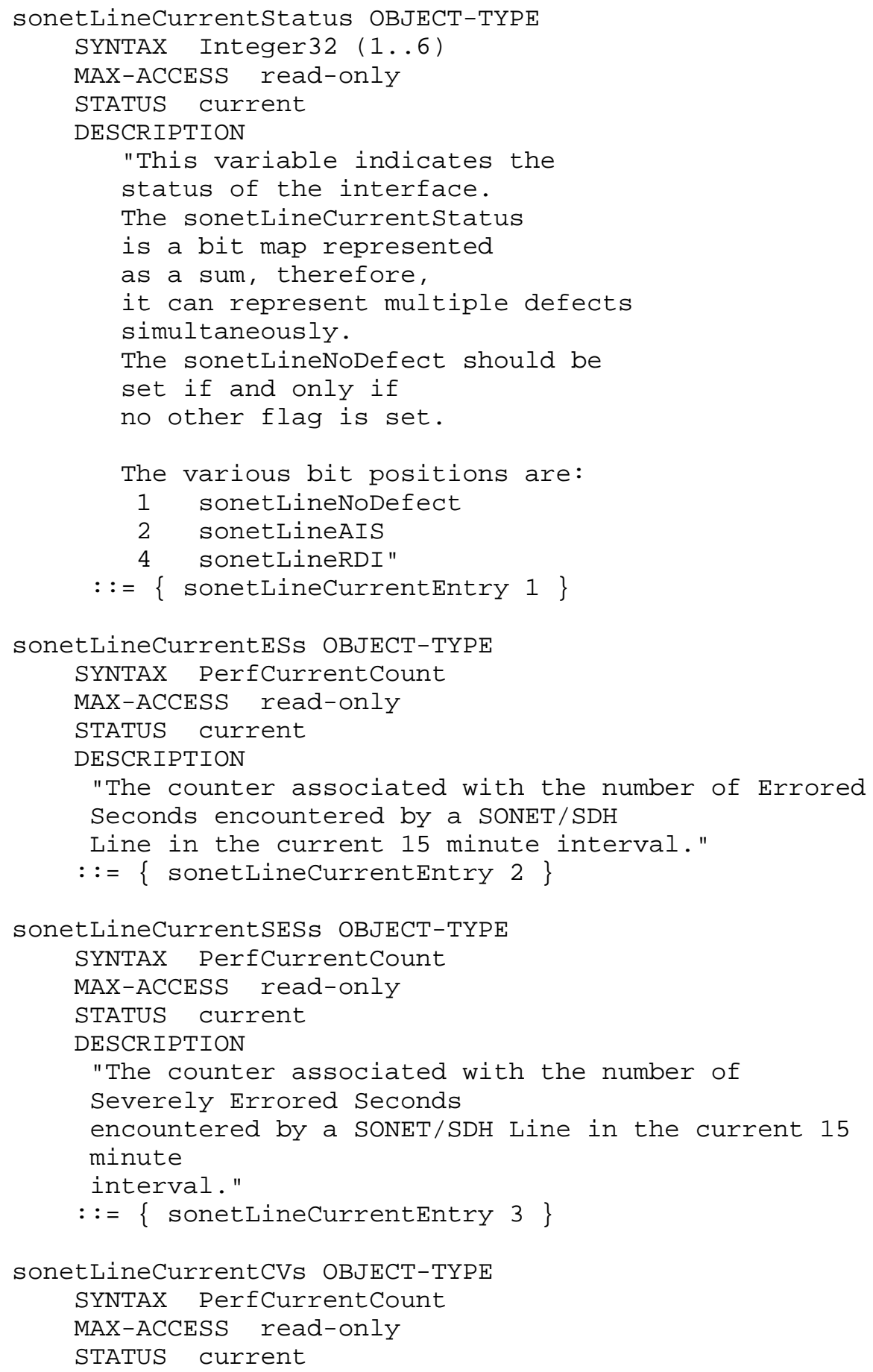


DESCRIPTION

"The counter associated with the number of Coding

Violations encountered by a

SONET/SDH Line in the current 15 minute interval." $::=\{$ sonetLineCurrentEntry 4$\}$

sonetLineCurrentUASs OBJECT-TYPE

SYNTAX PerfCurrentCount

MAX-ACCESS read-only

STATUS current

DESCRIPTION

"The counter associated with the number of

Unavailable Seconds

encountered by a SONET/SDH Line in the current 15

minute

interval."

$::=\{$ sonetLineCurrentEntry 5$\}$

-- the SONET/SDH Line Interval Table

-- The SONET/SDH Line Interval Table

- contains various statistics

-- collected by each system over a maximum

-- of the previous 24 hours of

-- operation. The past 24 hours may be broken into 96

-- completed 15 minute intervals.

-- A system is required to store at

-- least 4 completed 15 minute interval.

-- The default value is 32 intervals.

sonetLineIntervalTable OBJECT-TYPE

SYNTAX SEQUENCE OF SonetLineIntervalEntry

MAX-ACCESS not-accessible

STATUS current

DESCRIPTION

"The SONET/SDH Line Interval table."

$::=\{$ sonetLine 2$\}$

sonetLineIntervalEntry OBJECT-TYPE

SYNTAX SonetLineIntervalEntry

MAX-ACCESS not-accessible

STATUS current

DESCRIPTION

"An entry in the SONET/SDH Line Interval table."

INDEX $\{$ ifIndex, sonetLineIntervalNumber \}

$::=\{$ sonetLineIntervalTable 1$\}$ 


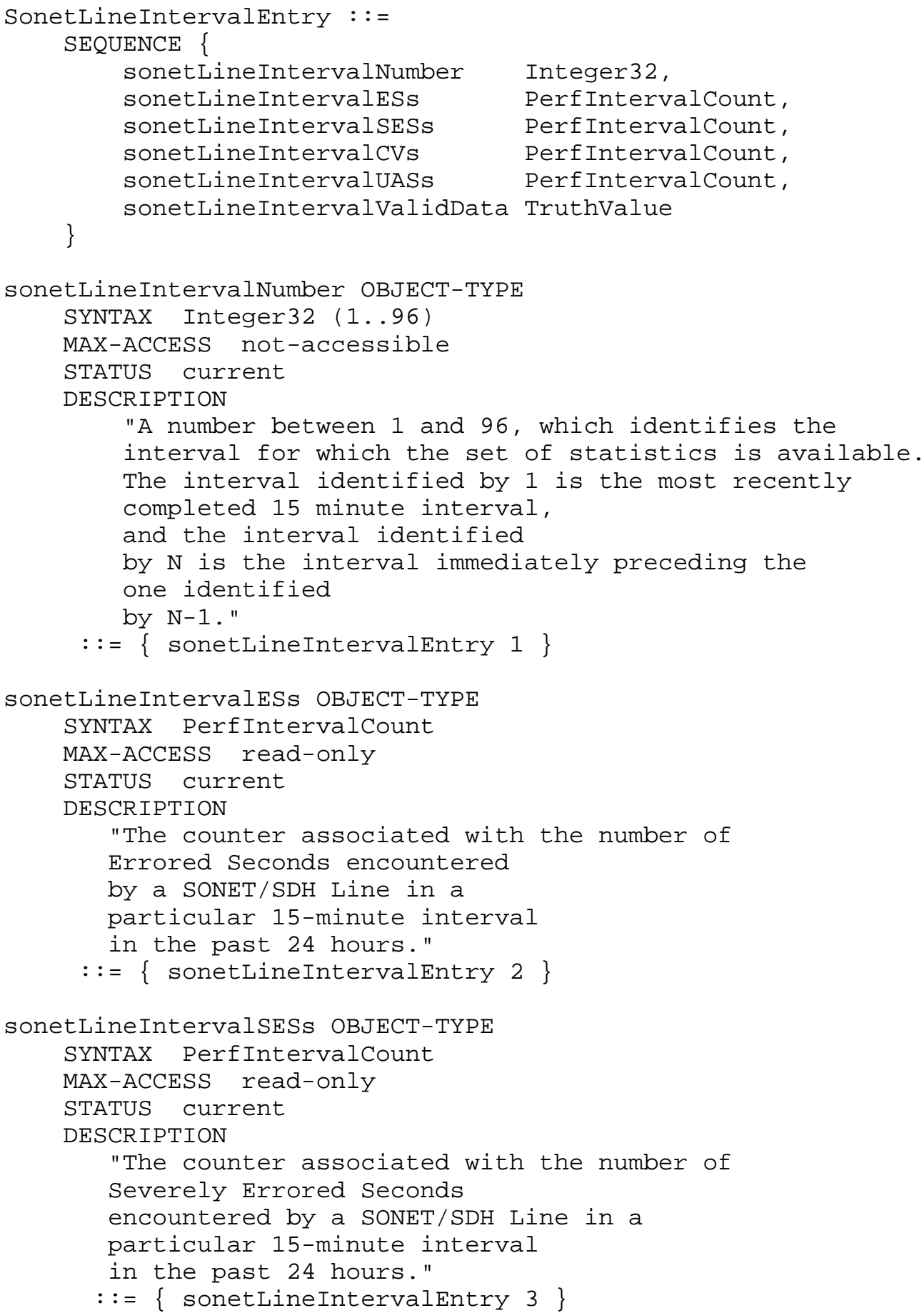

Tesink 


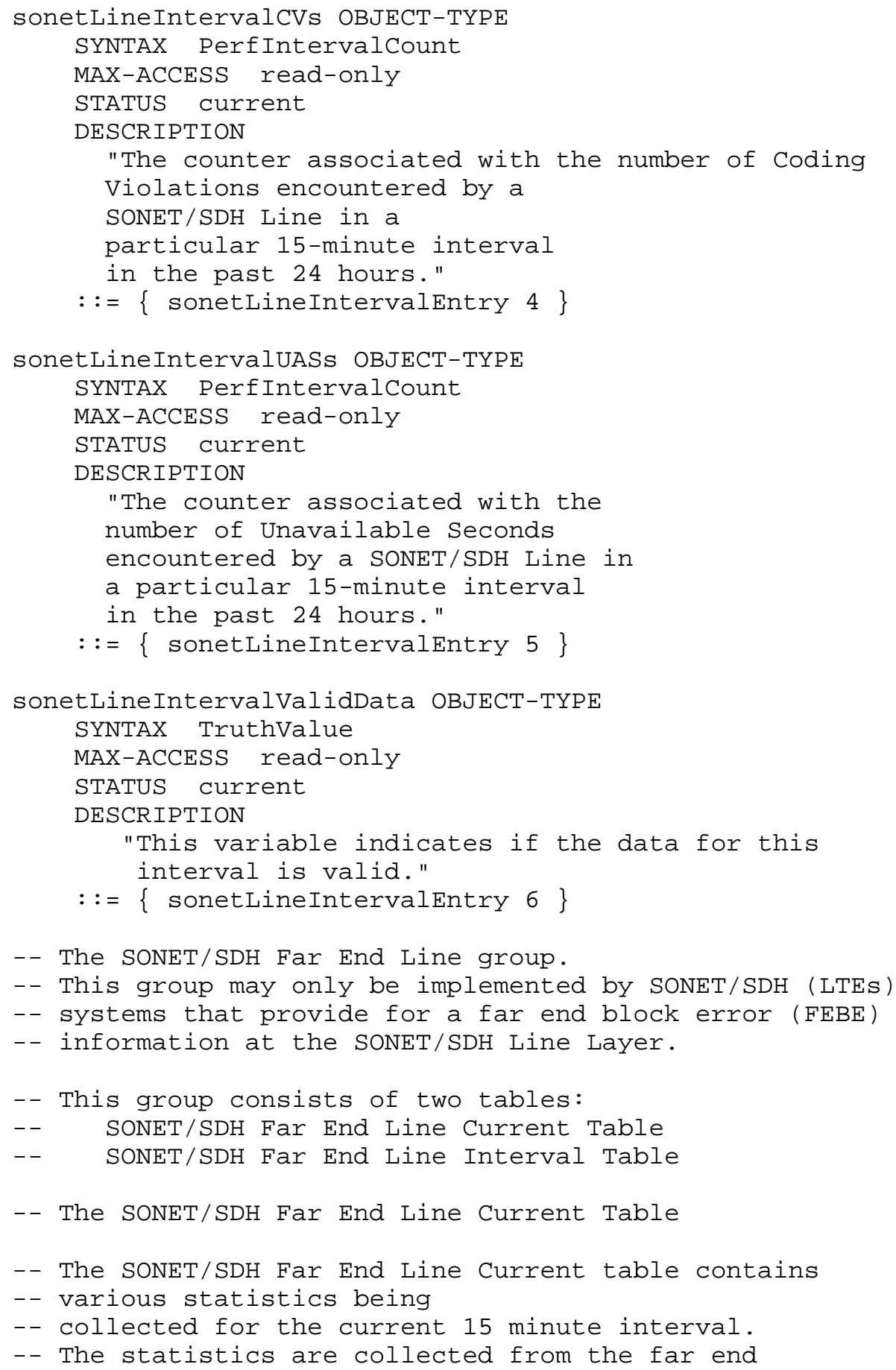




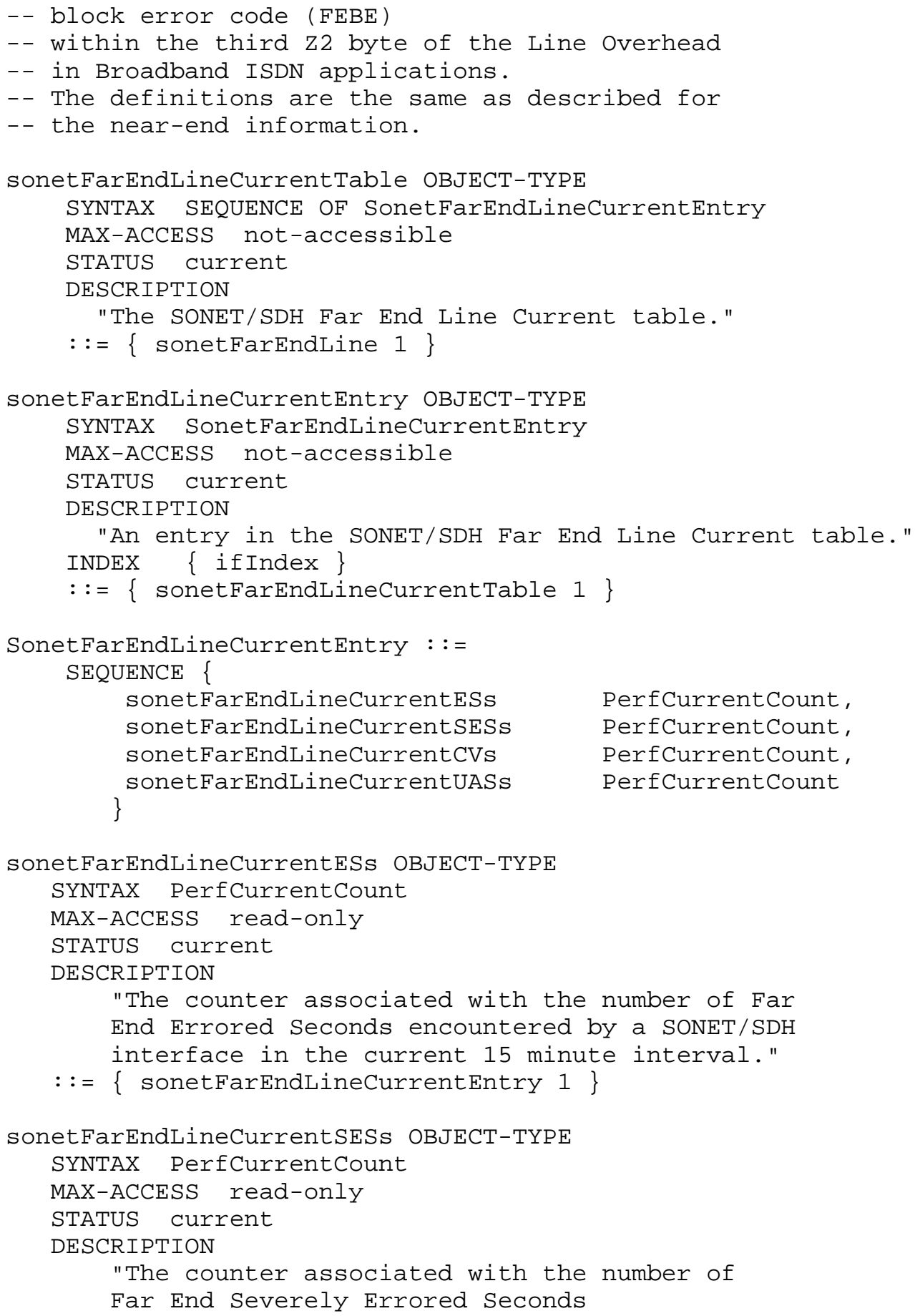

"The counter associated with the number of

Far End Severely Errored Seconds 


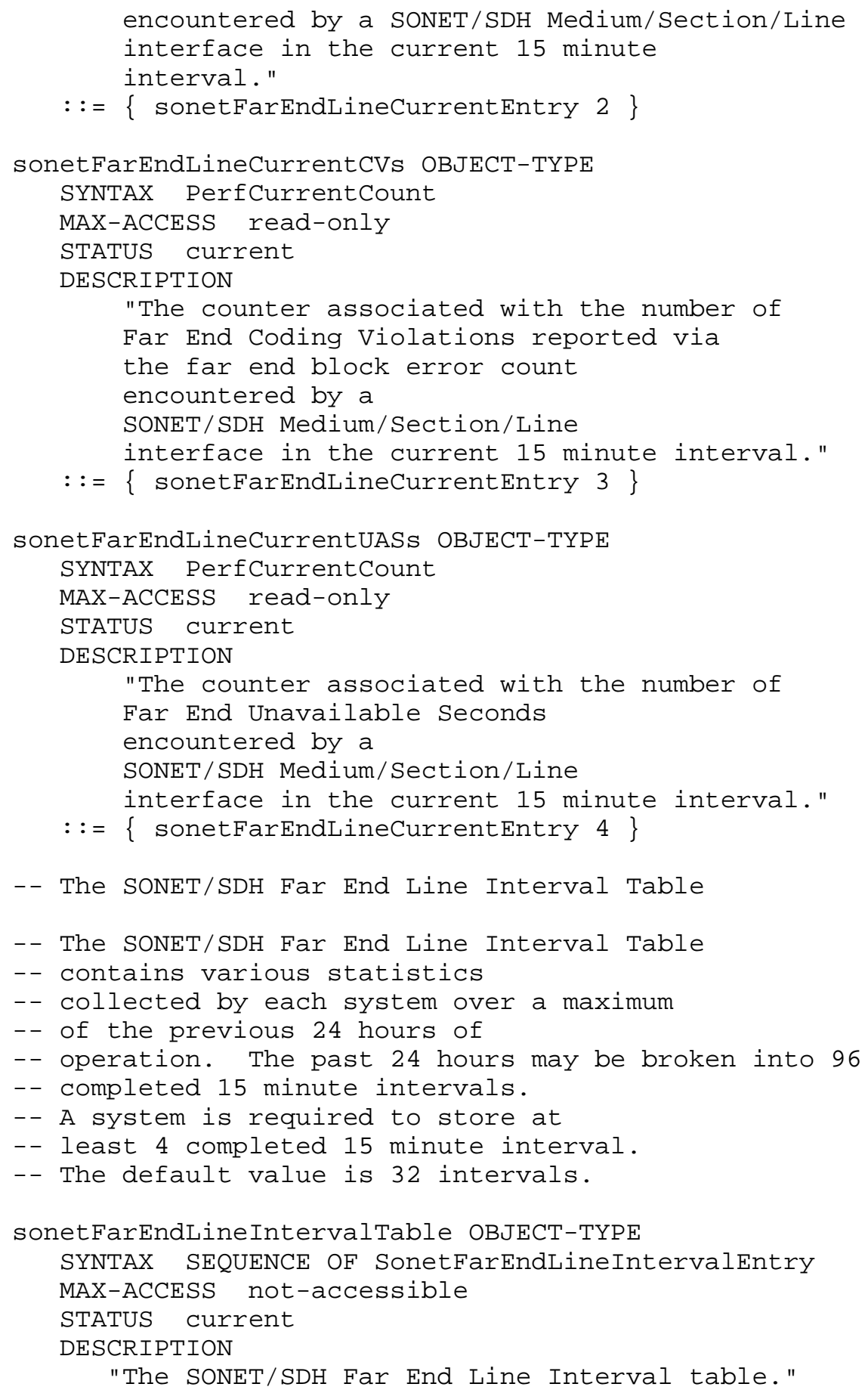


$::=\{$ sonetFarEndLine 2$\}$

sonetFarEndLineIntervalEntry OBJECT-TYPE

SYNTAX SonetFarEndLineIntervalEntry

MAX-ACCESS not-accessible

STATUS current

DESCRIPTION

"An entry in the SONET/SDH Far

End Line Interval table."

INDEX \{ ifIndex, sonetFarEndLineIntervalNumber \}

$::=\{$ sonetFarEndLineIntervalTable 1$\}$

SonetFarEndLineIntervalEntry : := SEQUENCE \{

sonetFarEndLineIntervalNumber

Integer32, sonetFarEndLineIntervalESs sonetFarEndLineIntervalSESS PerfIntervalcount, sonet $\mathrm{F}$ arEndLineIntervalCVs PerfIntervalcount, sonetFarEndLineIntervalUASs PerfIntervalcount, PerfIntervalcount, \} sonetFarEndLineIntervalValidData TruthValue

sonetFarEndLineIntervalNumber OBJECT-TYPE

SYNTAX Integer32 (1..96)

MAX-ACCESS not-accessible

STATUS current

DESCRIPTION

"A number between 1 and 96, which identifies the interval for which the set of statistics is available. The interval identified by 1 is the most recently completed 15 minute interval, and the interval identified

by $\mathrm{N}$ is the interval immediately preceding the one identified by $\mathrm{N}-1$."

$::=\{$ sonetFarEndLineIntervalEntry 1$\}$

sonetFarEndLineIntervalESs OBJECT-TYPE

SYNTAX PerfIntervalcount

MAX-ACCESS read-only

STATUS current

DESCRIPTION

"The counter associated with the number of

Far End Errored Seconds encountered

by a SONET/SDH Line

interface in a particular 15-minute interval

in the past 24 hours." 
$::=\{$ sonetFarEndLineIntervalEntry 2$\}$

sonetFarEndLineIntervalSESs OBJECT-TYPE

SYNTAX Perfintervalcount

MAX-ACCESS read-only

STATUS current

DESCRIPTION

"The counter associated with the number of

Far End Severely Errored Seconds

encountered by a SONET/SDH Line

interface in a particular 15-minute interval

in the past 24 hours."

$::=\{$ sonetFarEndLineIntervalEntry 3$\}$

sonetFarEndLineIntervalCVs OBJECT-TYPE

SYNTAX Perfintervalcount

MAX-ACCESS read-only

STATUS current

DESCRIPTION

"The counter associated with the number of

Far End Coding Violations reported via

the far end block error count

encountered by a

SONET/SDH Line

interface in a particular 15-minute interval

in the past 24 hours."

$::=\{$ sonetFarEndLineIntervalEntry 4$\}$

sonetFarEndLineIntervalUASs OBJECT-TYPE

SYNTAX PerfIntervalcount

MAX-ACCESS read-only

STATUS current

DESCRIPTION

"The counter associated with the number of

Far End Unavailable Seconds

encountered by a

SONET/SDH Line

interface in a particular 15-minute interval

in the past 24 hours."

$::=\{$ sonetFarEndLineIntervalEntry 5$\}$

sonetFarEndLineIntervalValidData OBJECT-TYPE

SYNTAX TruthValue

MAX-ACCESS read-only

STATUS current

DESCRIPTION

"This variable indicates if the data for this interval is valid." 
$::=\{$ sonetFarEndLineIntervalEntry 6$\}$

-- the SONET/SDH Path group

-- this group consists of 2 tables:

-- - the SONET/SDH Path Current Table

- - the SONET/SDH Path Interval Table

-- the SONET/SDH Path Current Table

-- The SONET/SDH Path

-- current table contains various statistics

-- being collected for the current 15 minute interval.

sonetPathCurrentTable OBJECT-TYPE

SYNTAX SEQUENCE OF SonetPathCurrentEntry

MAX-ACCESS not-accessible

STATUS current

DESCRIPTION

"The SONET/SDH Path Current table."

$::=\{$ sonetPath 1$\}$

sonetPathCurrentEntry OBJECT-TYPE

SYNTAX SonetPathCurrentEntry

MAX-ACCESS not-accessible

STATUS current

DESCRIPTION

"An entry in the SONET/SDH Path Current table." INDEX \{ifIndex $\}$

$::=\{$ sonetPathCurrentTable 1$\}$

SonetPathCurrentEntry : := SEQUENCE \{

sonetPathCurrentWidth INTEGER, sonetPathCurrentStatus Integer32, sonetPathCurrentESs PerfCurrentCount, sonetPathCurrentSESs PerfCurrentCount, sonetPathCurrentCVs PerfCurrentCount, sonetPathCurrentUASs PerfCurrentCount

\}

sonetPathCurrentWidth OBJECT-TYPE

SYNTAX INTEGER \{

sts1 (1),

sts 3CSTM1 (2),

sts 12 CSTM4 (3),

sts $24 \mathrm{C}(4)$,

sts 48 CSTM16 (5),

Tesink

Standards Track

[Page 37] 


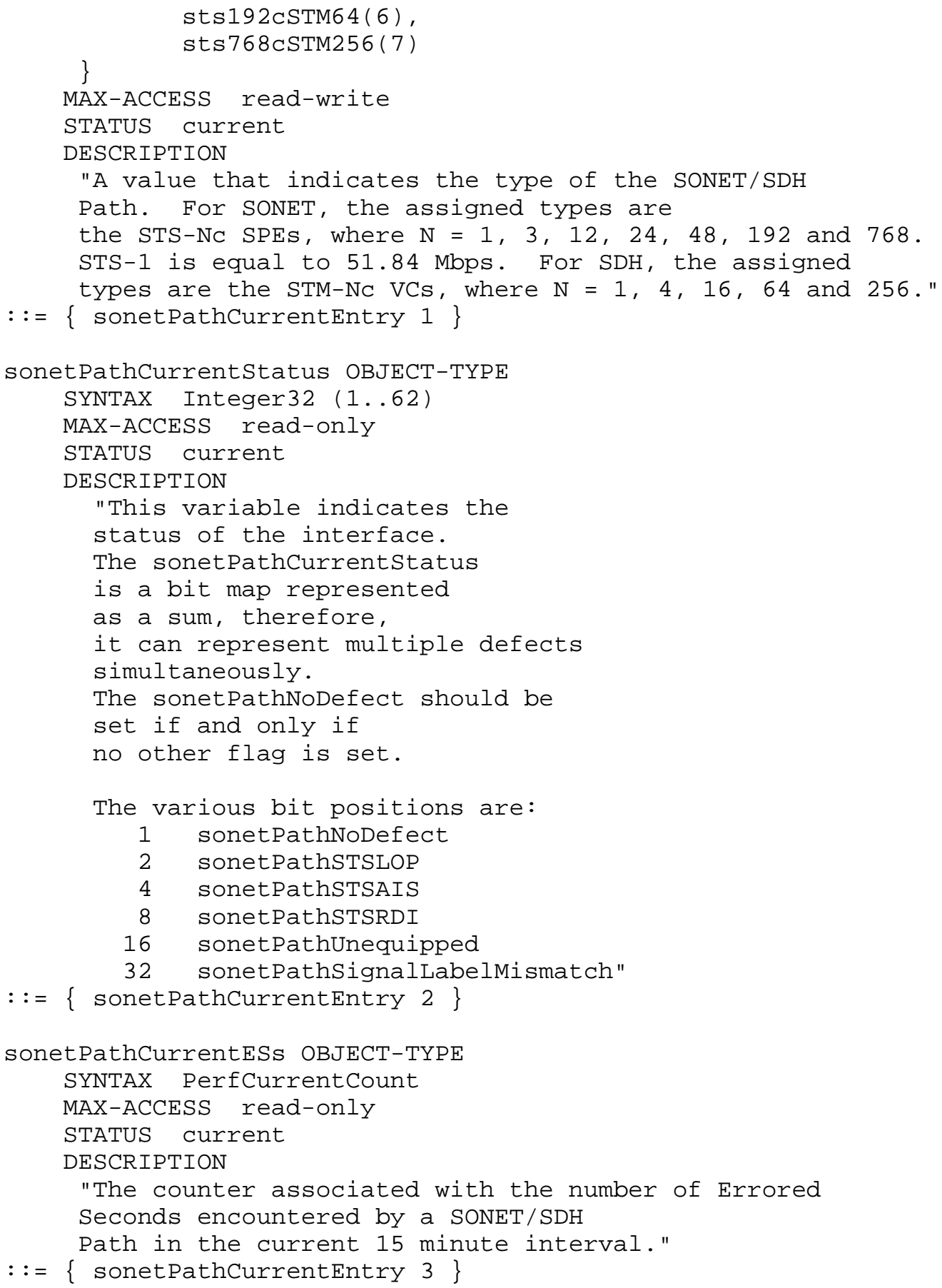




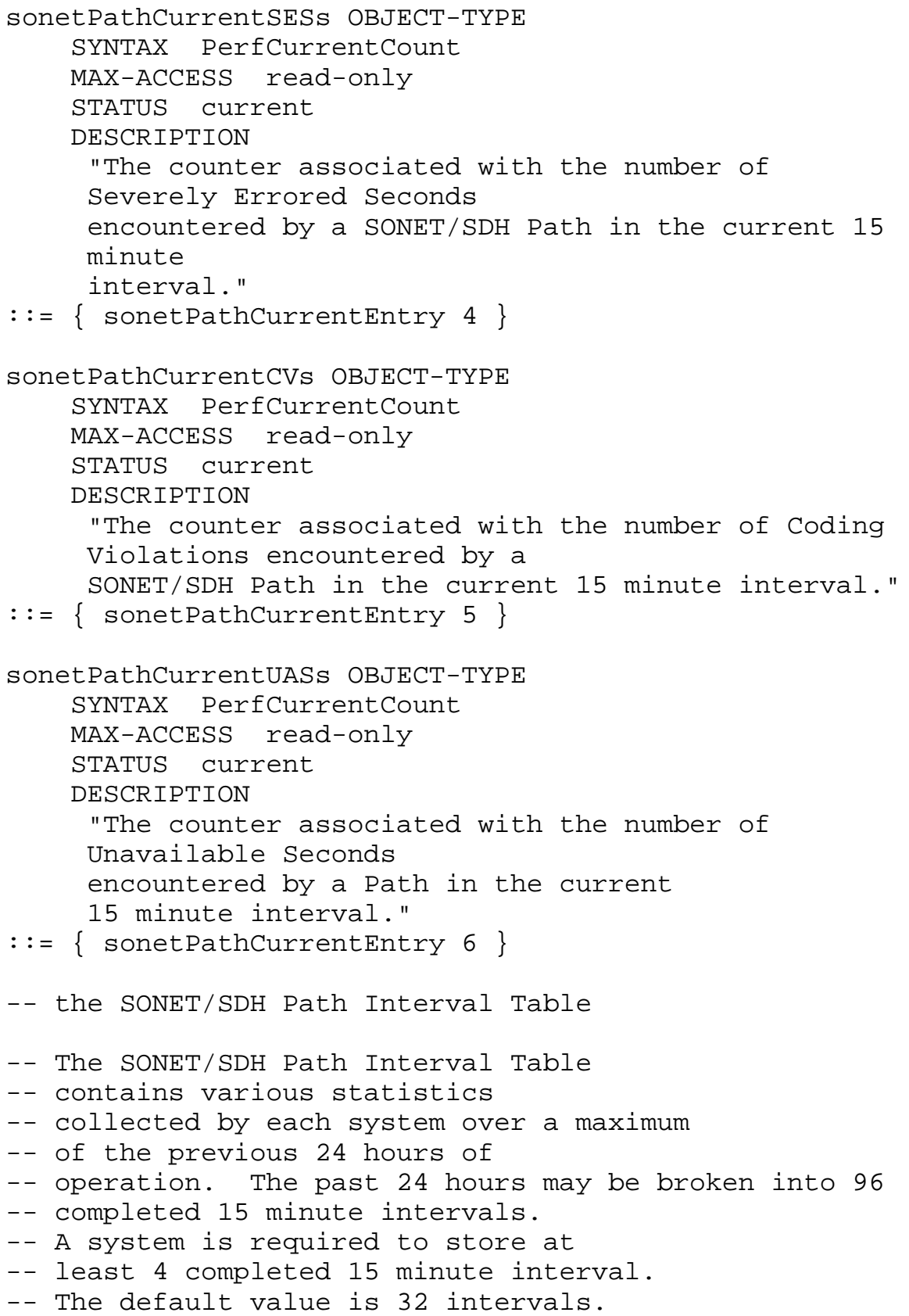




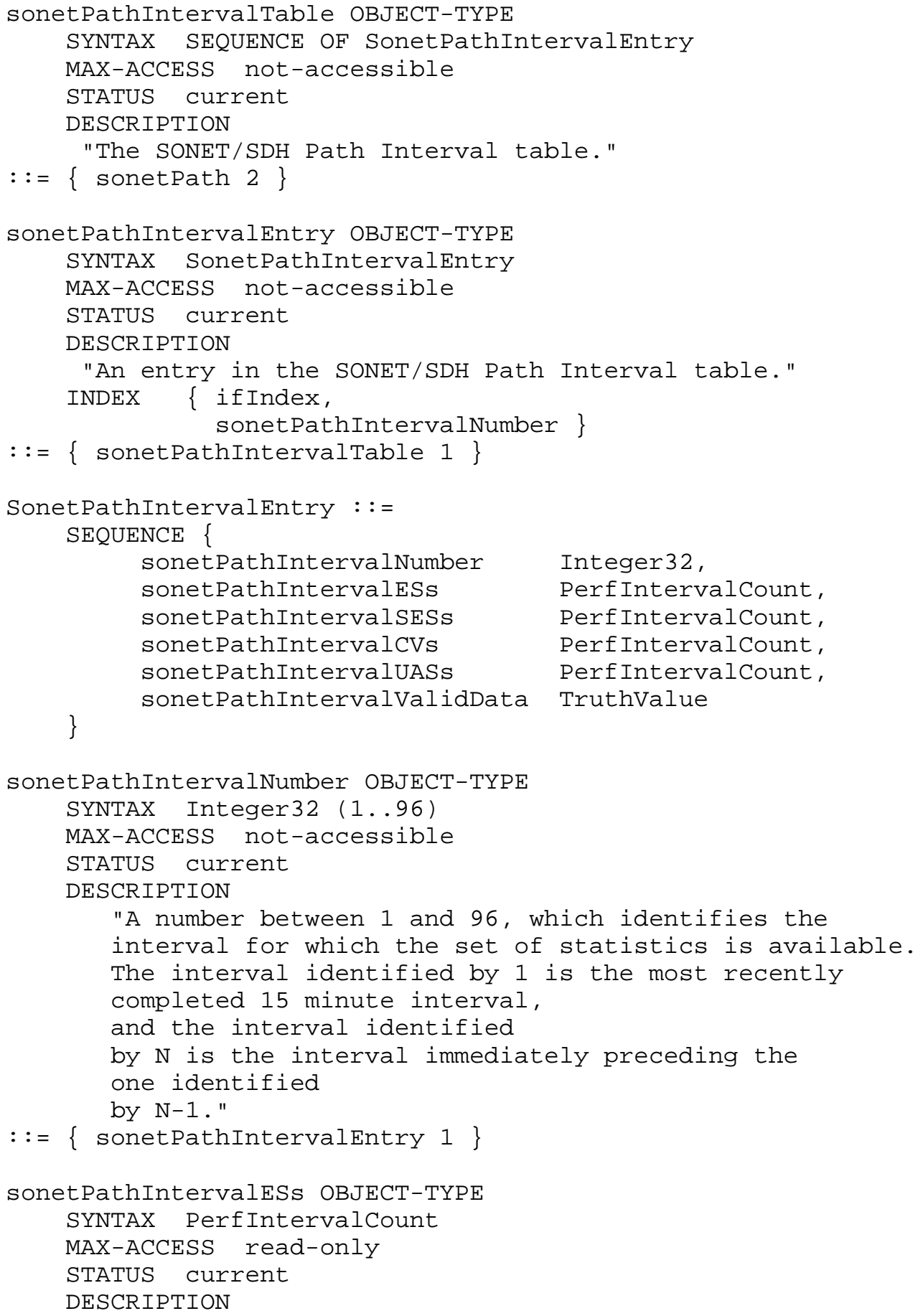




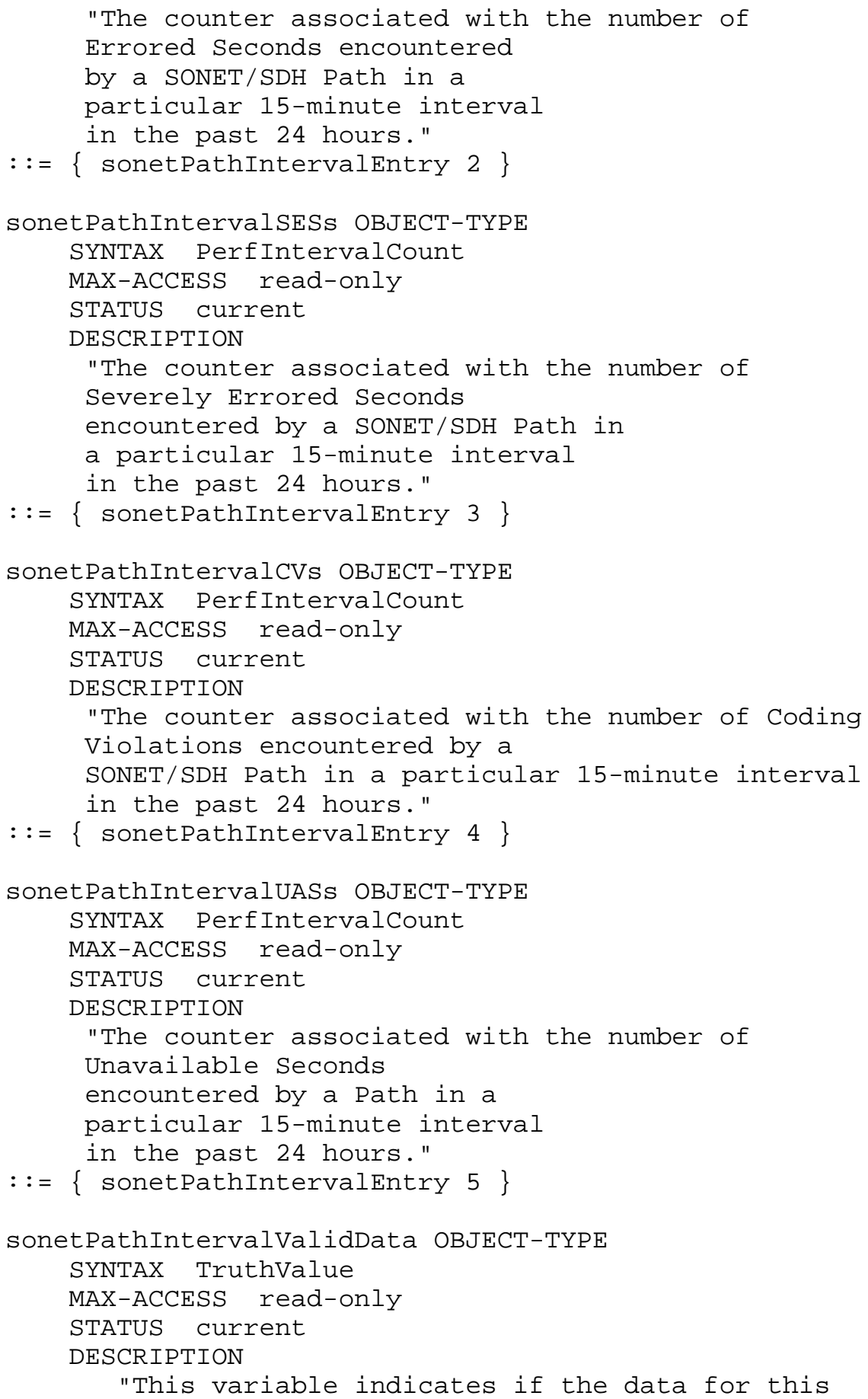




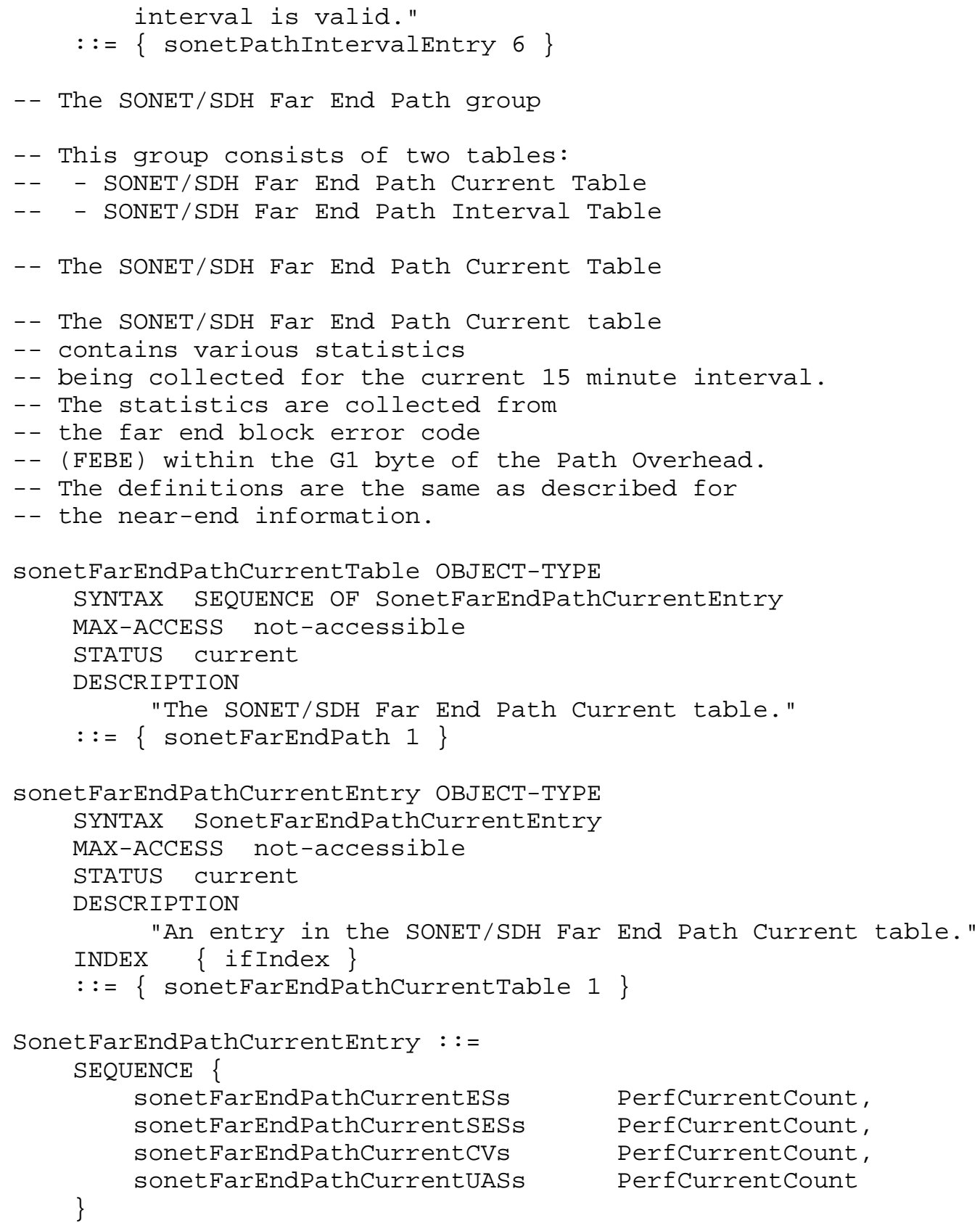




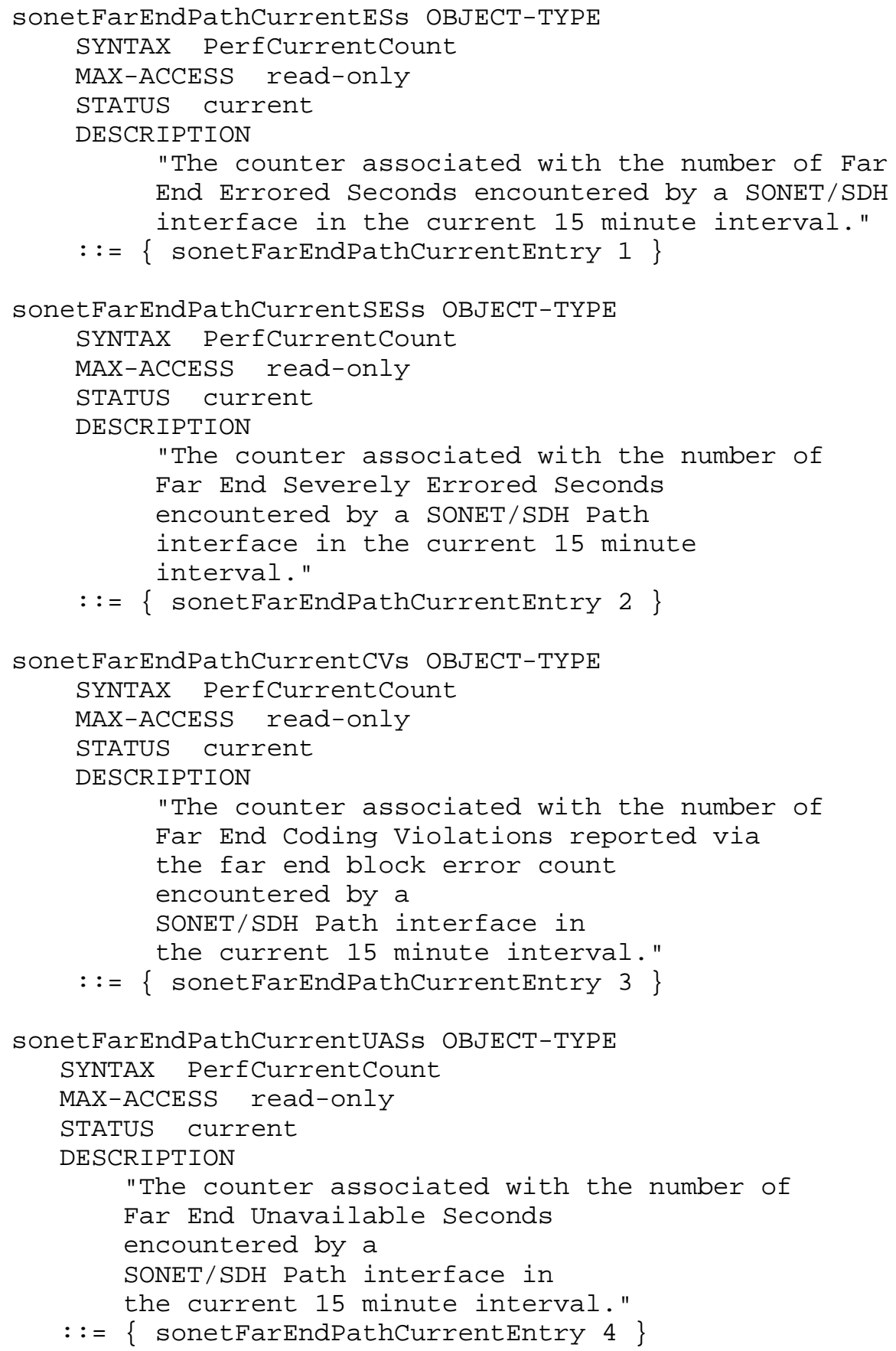


-- The SONET/SDH Far End Path Interval Table

-- The SONET/SDH Far End Path Interval Table

-- contains various statistics

-- collected by each system over a maximum

-- of the previous 24 hours of

-- operation. The past 24 hours may be broken into 96

-- completed 15 minute intervals.

-- A system is required to store at

-- least 4 completed 15 minute interval.

-- The default value is 32 intervals.

sonetFarEndPathIntervalTable OBJECT-TYPE

SYNTAX SEQUENCE OF SonetFarEndPathIntervalEntry

MAX-ACCESS not-accessible

STATUS current

DESCRIPTION

"The SONET/SDH Far End Path Interval table."

$::=\{$ sonetFarEndPath 2$\}$

sonetFarEndPathIntervalEntry OBJECT-TYPE

SYNTAX SonetFarEndPathIntervalEntry

MAX-ACCESS not-accessible

STATUS current

DESCRIPTION

"An entry in the SONET/SDH Far

End Path Interval table."

INDEX $\{$ ifIndex, sonetFarEndPathIntervalNumber \}

$::=\{$ sonetFarEndPathIntervalTable 1$\}$

SonetFarEndPathIntervalEntry : := SEQUENCE \{

sonetFarEndPathIntervalNumber Integer32,

sonetFarEndPathIntervalESs

sonetFarEndPathIntervalSESS

sonetFarEndPathIntervalCVs

sonetFarEndPathIntervalUASs

PerfIntervalcount,

Perfintervalcount, PerfIntervalcount, sonetFarEndPathIntervalValidData TruthValue

\}

sonetFarEndPathIntervalNumber OBJECT-TYPE

SYNTAX Integer32 (1..96)

MAX-ACCESS not-accessible

STATUS current

DESCRIPTION

"A number between 1 and 96, which identifies the

interval for which the set of statistics is available. 
The interval identified by 1 is the most recently completed 15 minute interval, and the interval identified

by $\mathrm{N}$ is the interval immediately preceding the one identified by $\mathrm{N}-1 . "$

$::=\{$ sonetFarEndPathIntervalEntry 1$\}$

sonetFarEndPathIntervalESs OBJECT-TYPE

SYNTAX PerfIntervalcount

MAX-ACCESS read-only

STATUS current

DESCRIPTION

"The counter associated with the number of

Far End Errored Seconds encountered

by a SONET/SDH Path interface in a

particular 15-minute interval

in the past 24 hours."

$::=\{$ sonetFarEndPathIntervalEntry 2$\}$

sonetFarEndPathIntervalSESS OBJECT-TYPE

SYNTAX PerfIntervalcount

MAX-ACCESS read-only

STATUS current

DESCRIPTION

"The counter associated with the number of

Far End Severely Errored Seconds

encountered by a SONET/SDH Path interface

in a particular 15-minute interval

in the past 24 hours."

$::=\{$ sonetFarEndPathIntervalEntry 3$\}$

sonetFarEndPathIntervalCVs OBJECT-TYPE

SYNTAX Perfintervalcount

MAX-ACCESS read-only

STATUS current

DESCRIPTION

"The counter associated with the number of

Far End Coding Violations reported via

the far end block error count

encountered by a

SONET/SDH Path interface

in a particular 15-minute interval

in the past 24 hours."

$::=\{$ sonetFarEndPathIntervalEntry 4$\}$ 


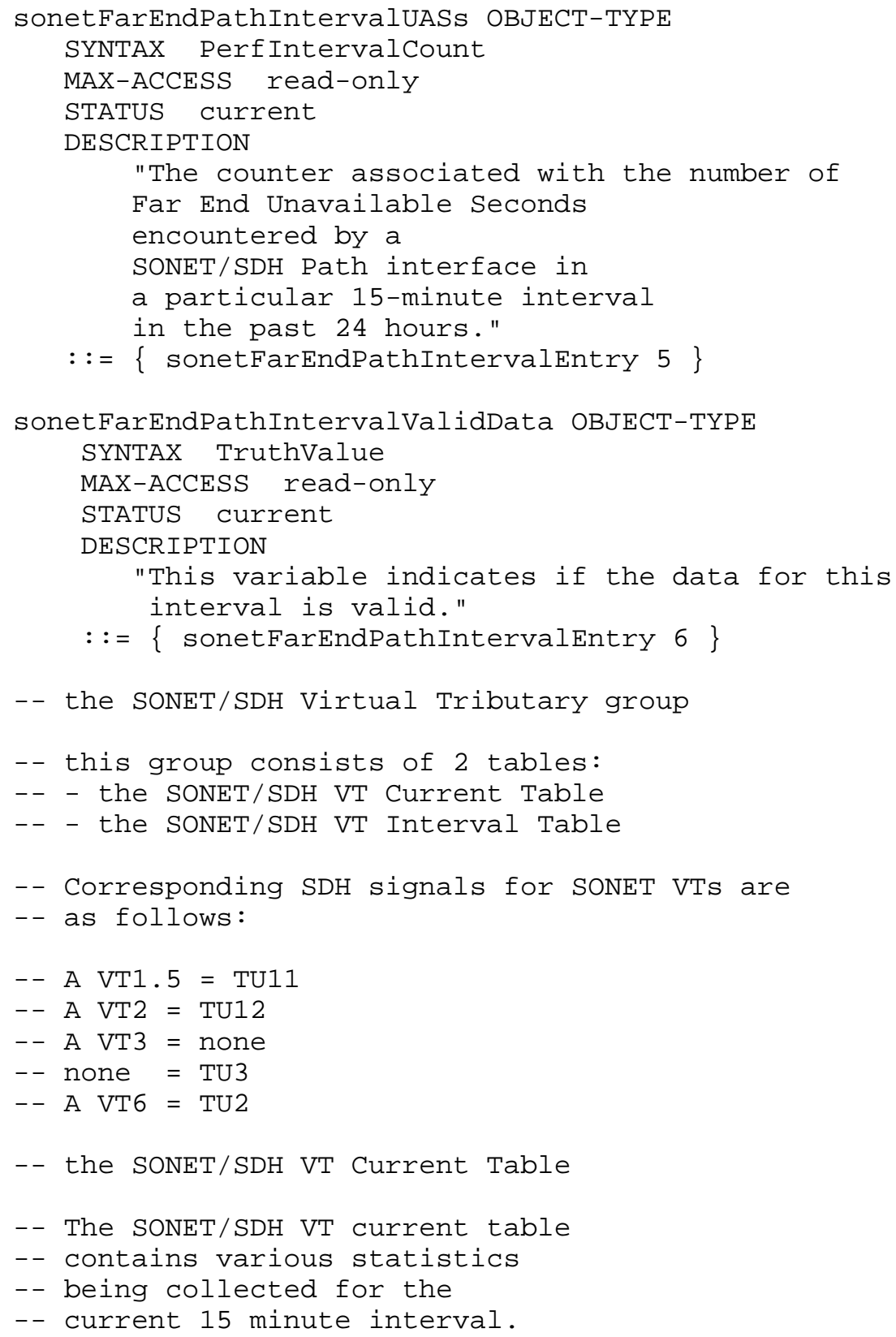




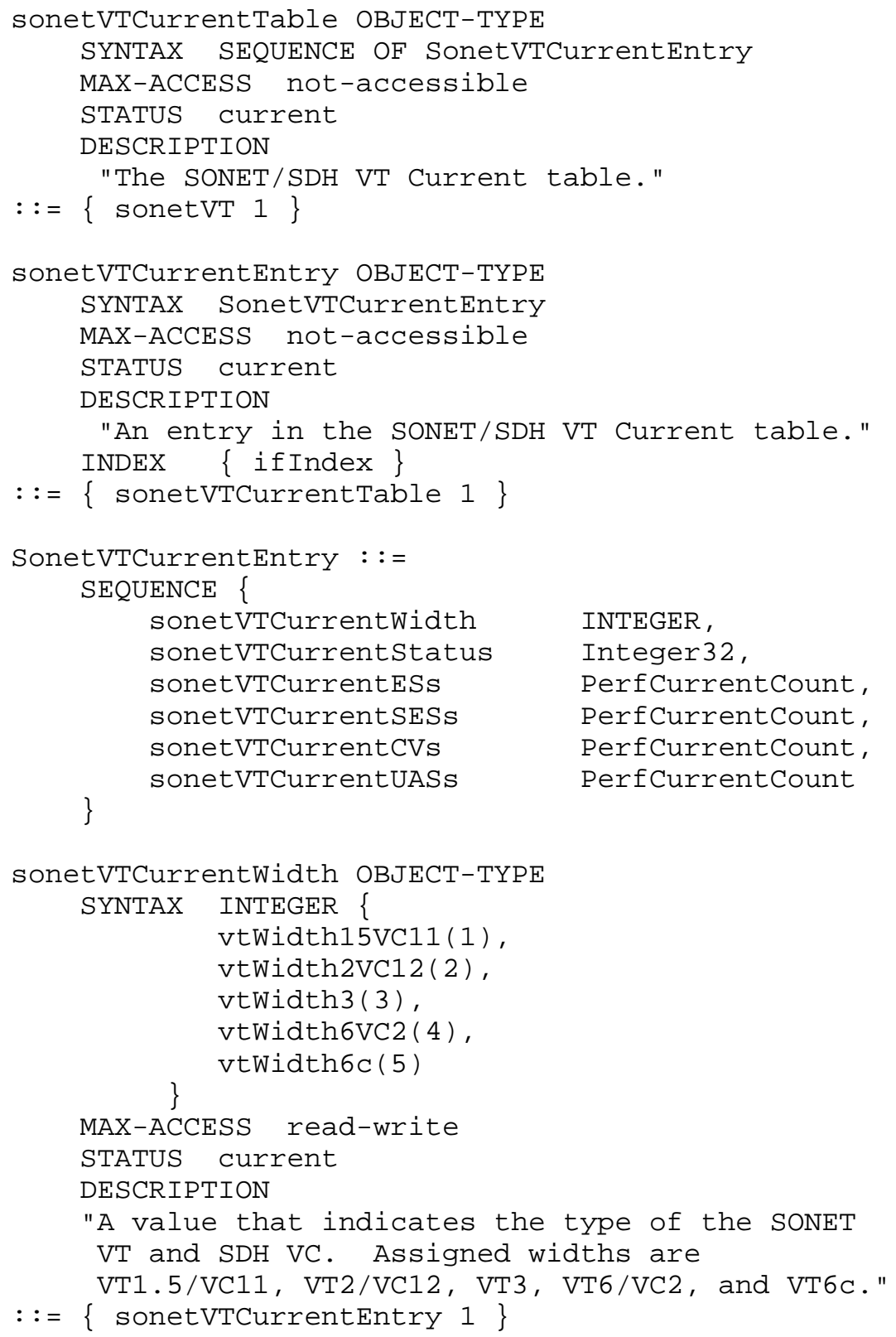




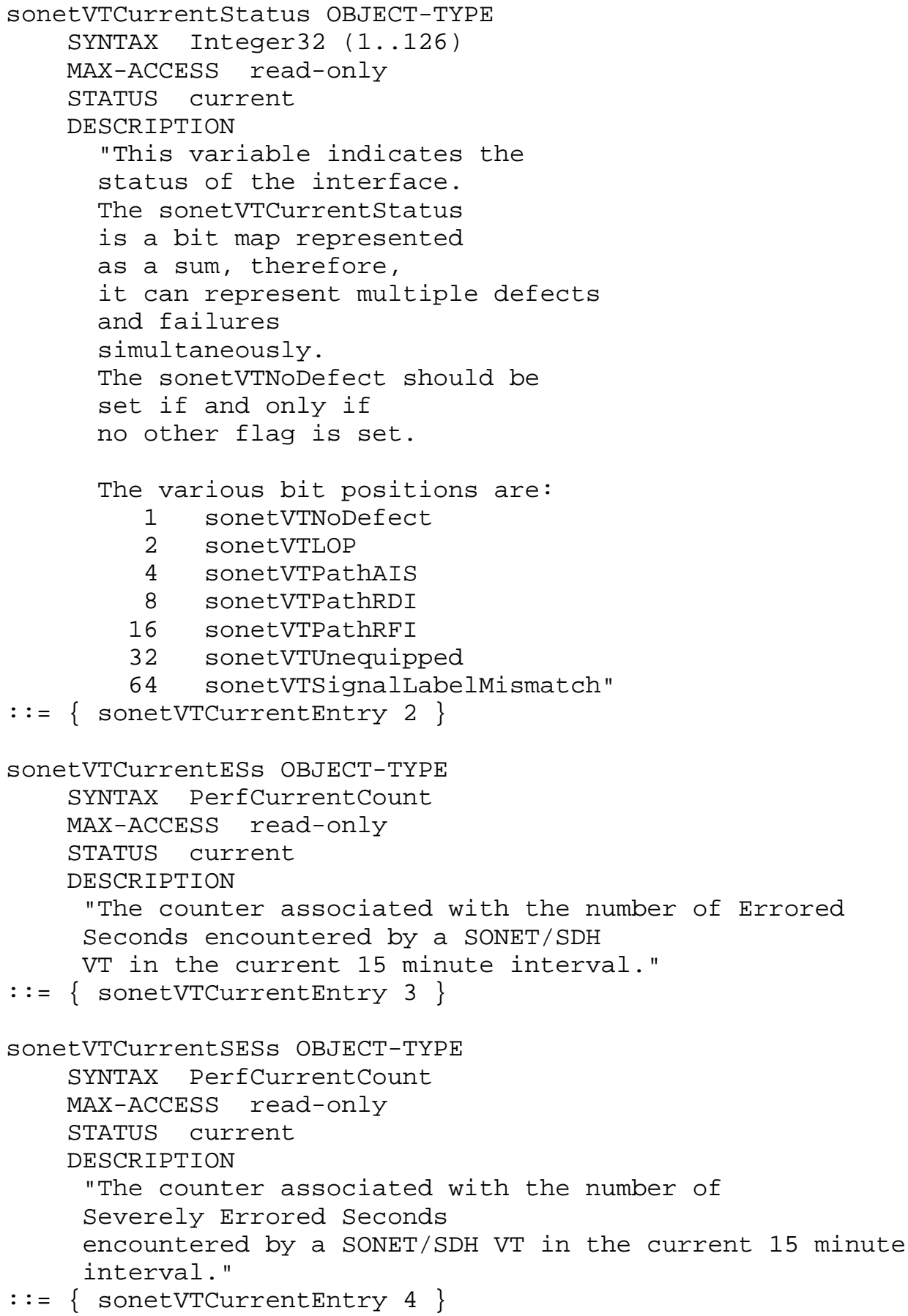




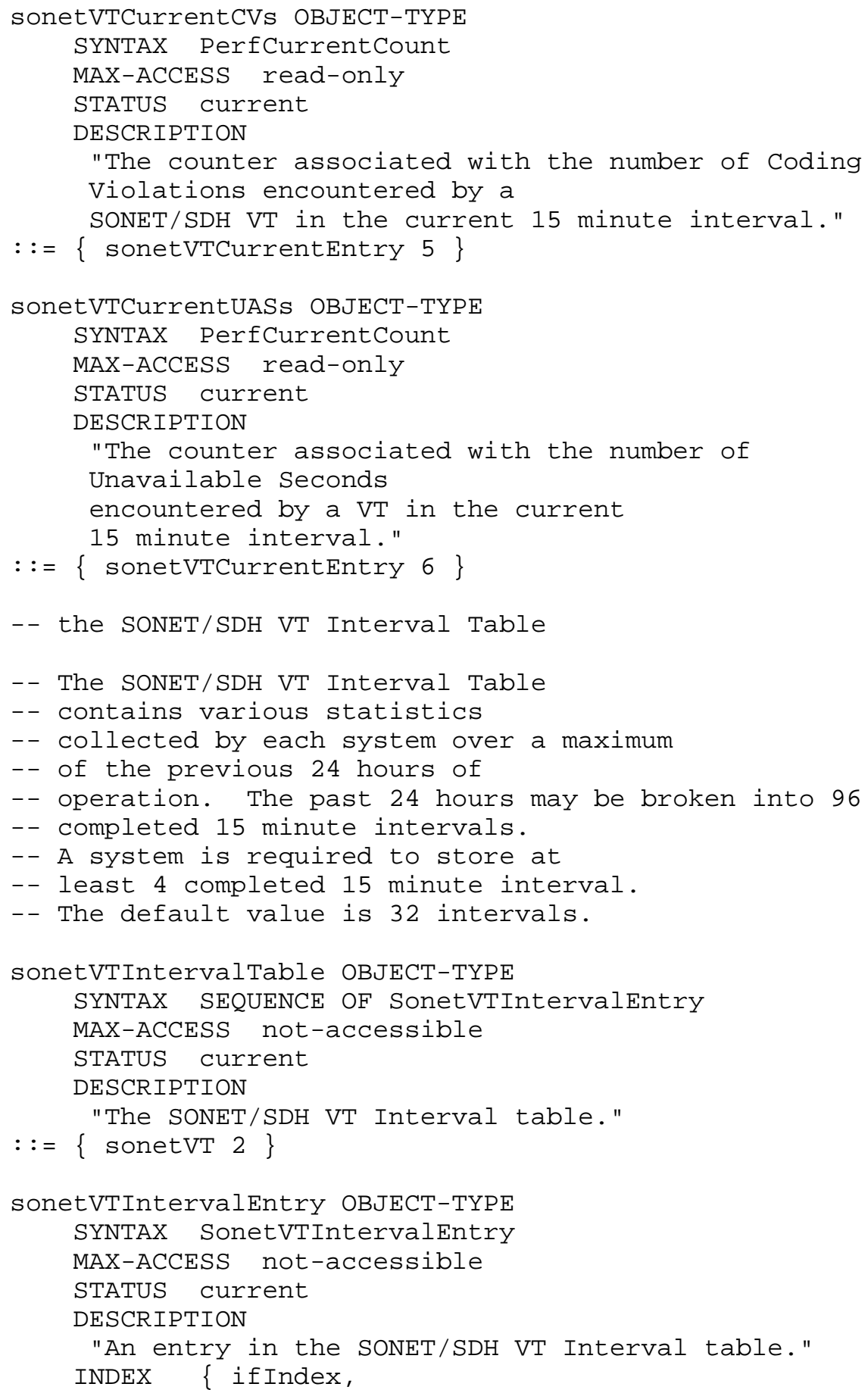




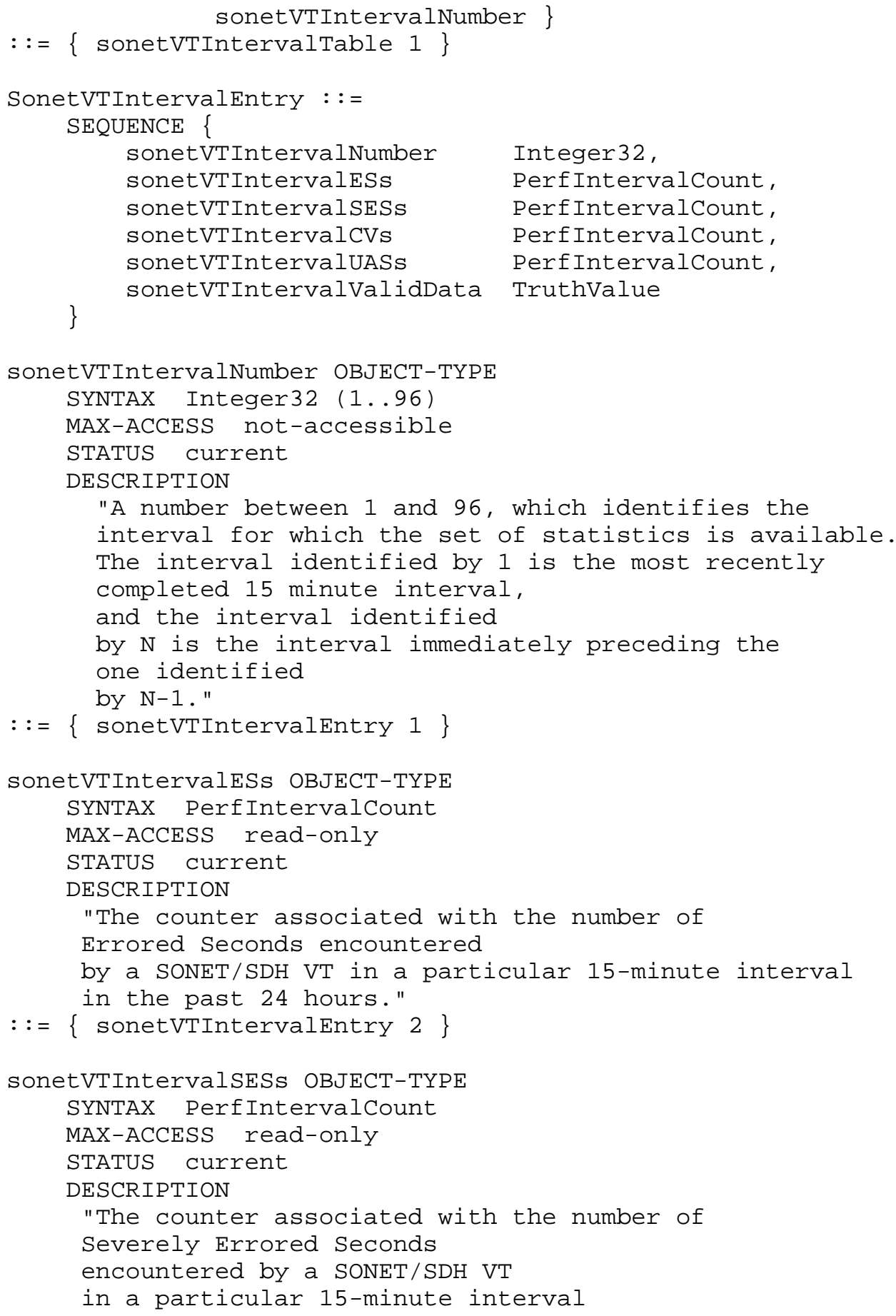




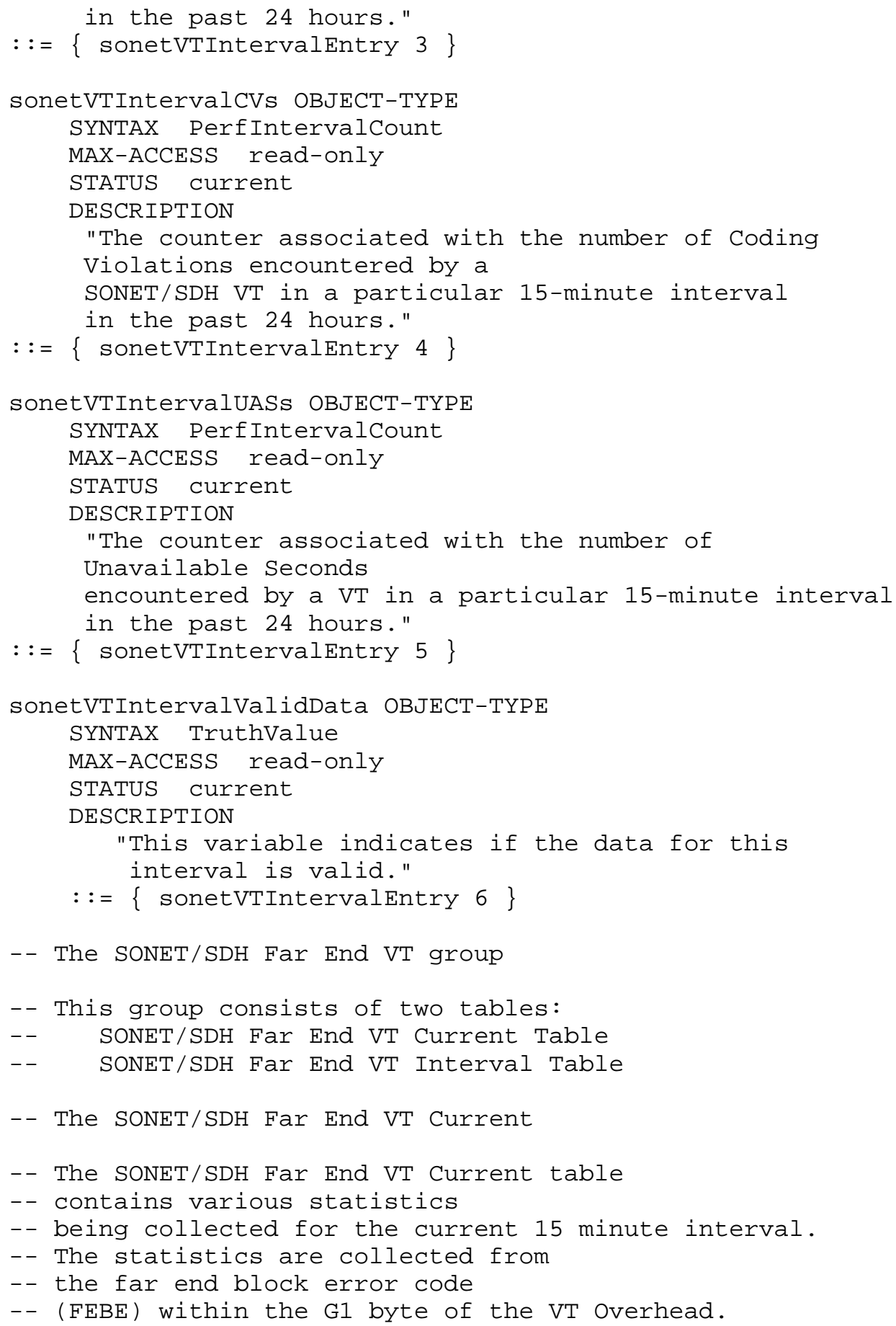




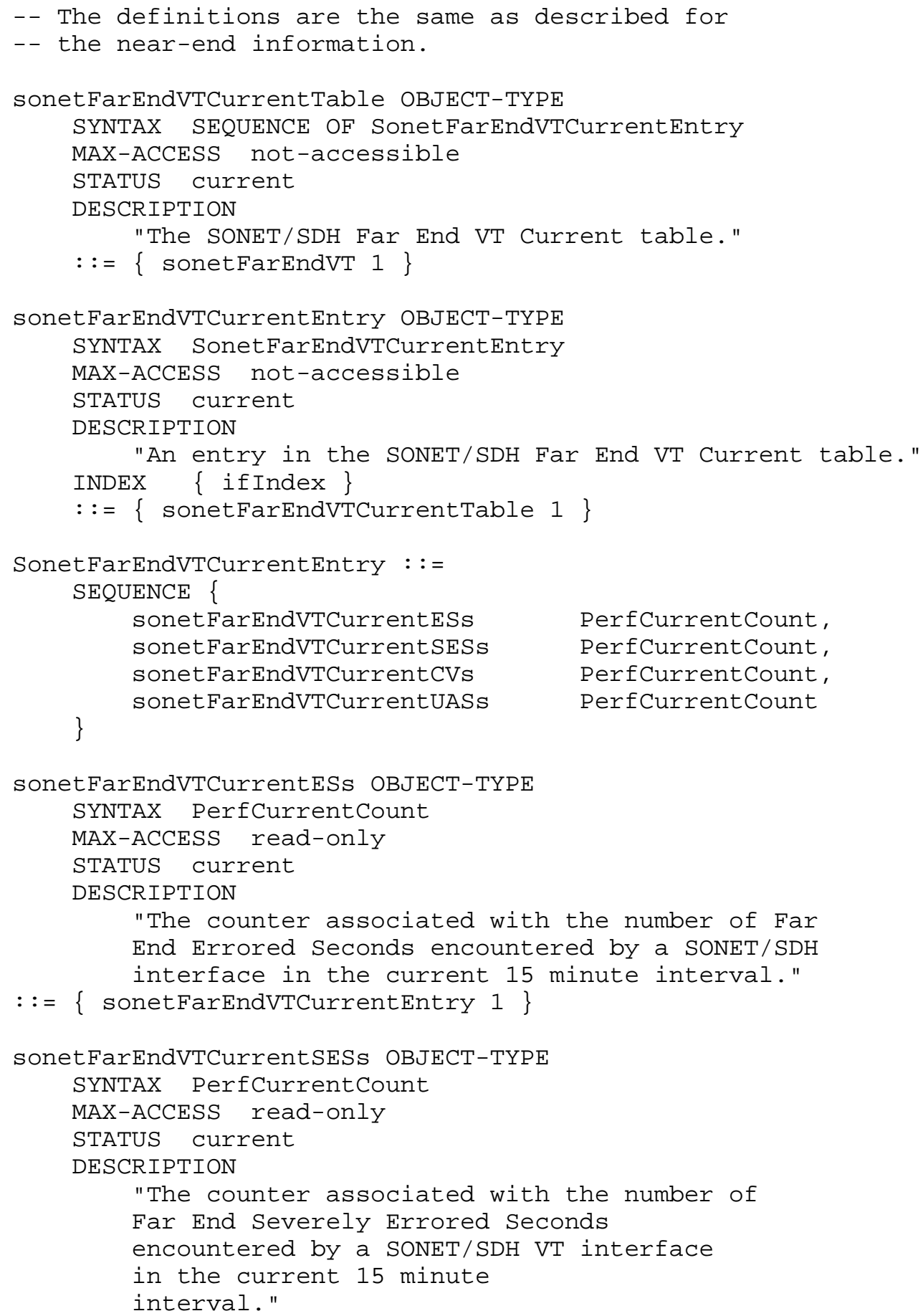


$::=\{$ sonetFarEndVTCurrentEntry 2$\}$

sonetFarEndVTCurrentCVs OBJECT-TYPE SYNTAX PerfCurrentCount

MAX-ACCESS read-only

STATUS current

DESCRIPTION

"The counter associated with the number of

Far End Coding Violations reported via

the far end block error count

encountered by a

SONET/SDH VT interface

in the current 15 minute interval."

$::=\{$ sonetFarEndVTCurrentEntry 3$\}$

sonetFarEndVTCurrentUASs OBJECT-TYPE

SYNTAX PerfCurrentCount

MAX-ACCESS read-only

STATUS current

DESCRIPTION

"The counter associated with the number of

Far End Unavailable Seconds

encountered by a

SONET/SDH VT interface

in the current 15 minute interval."

$::=\{$ sonetFarEndVTCurrentEntry 4$\}$

-- The SONET/SDH Far End VT Interval Table

-- The SONET/SDH Far End VT Interval Table

-- contains various statistics

-- collected by each system over a maximum

-- of the previous 24 hours of

-- operation. The past 24 hours may be broken into 96

-- completed 15 minute intervals.

-- A system is required to store at

-- least 4 completed 15 minute interval.

-- The default value is 32 intervals.

sonetFarEndVTIntervalTable OBJECT-TYPE

SYNTAX SEQUENCE OF SonetFarEndVTIntervalEntry

MAX-ACCESS not-accessible

STATUS current

DESCRIPTION

"The SONET/SDH Far End VT Interval table."

$::=\{$ sonetFarEndVT 2$\}$ 


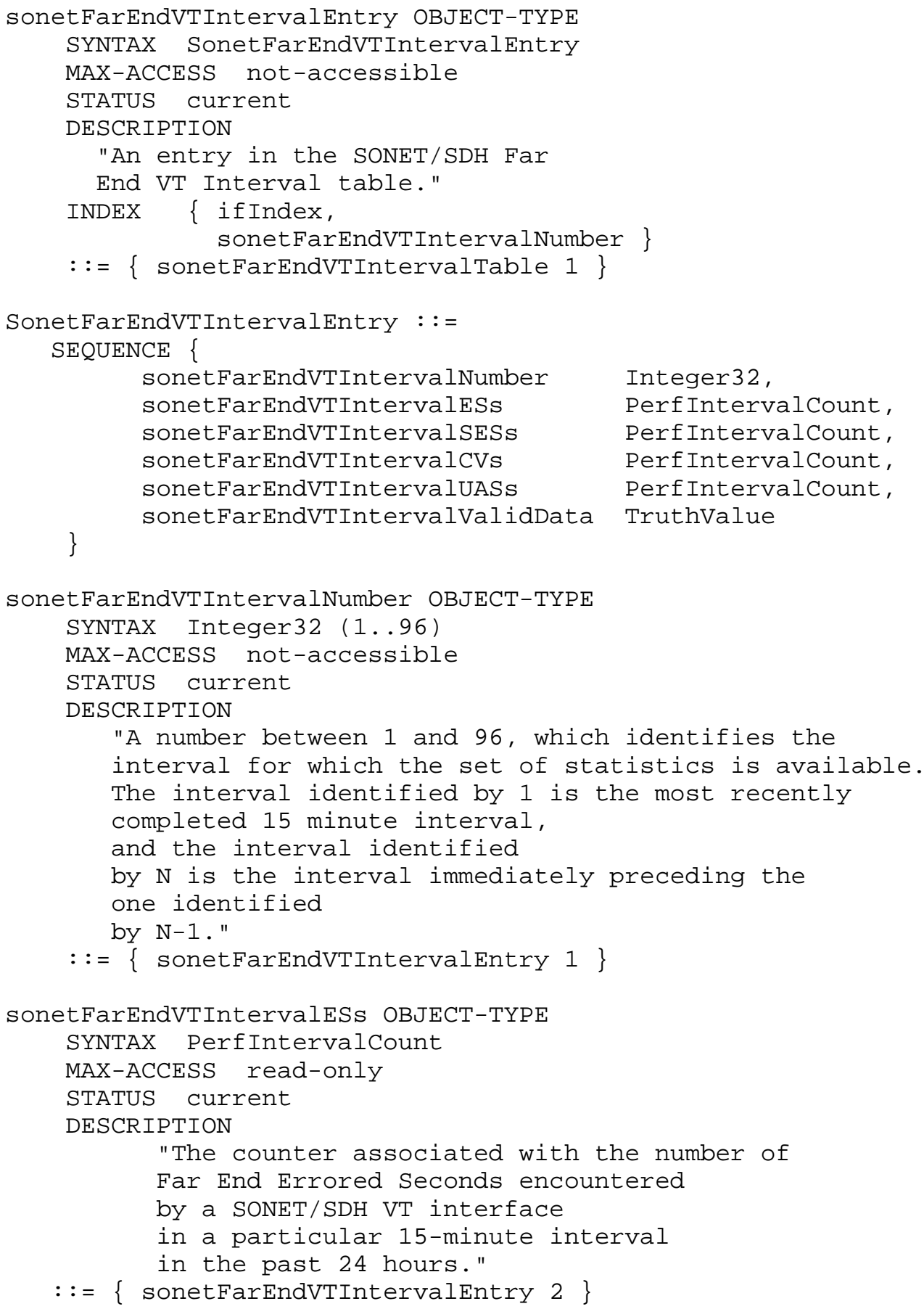




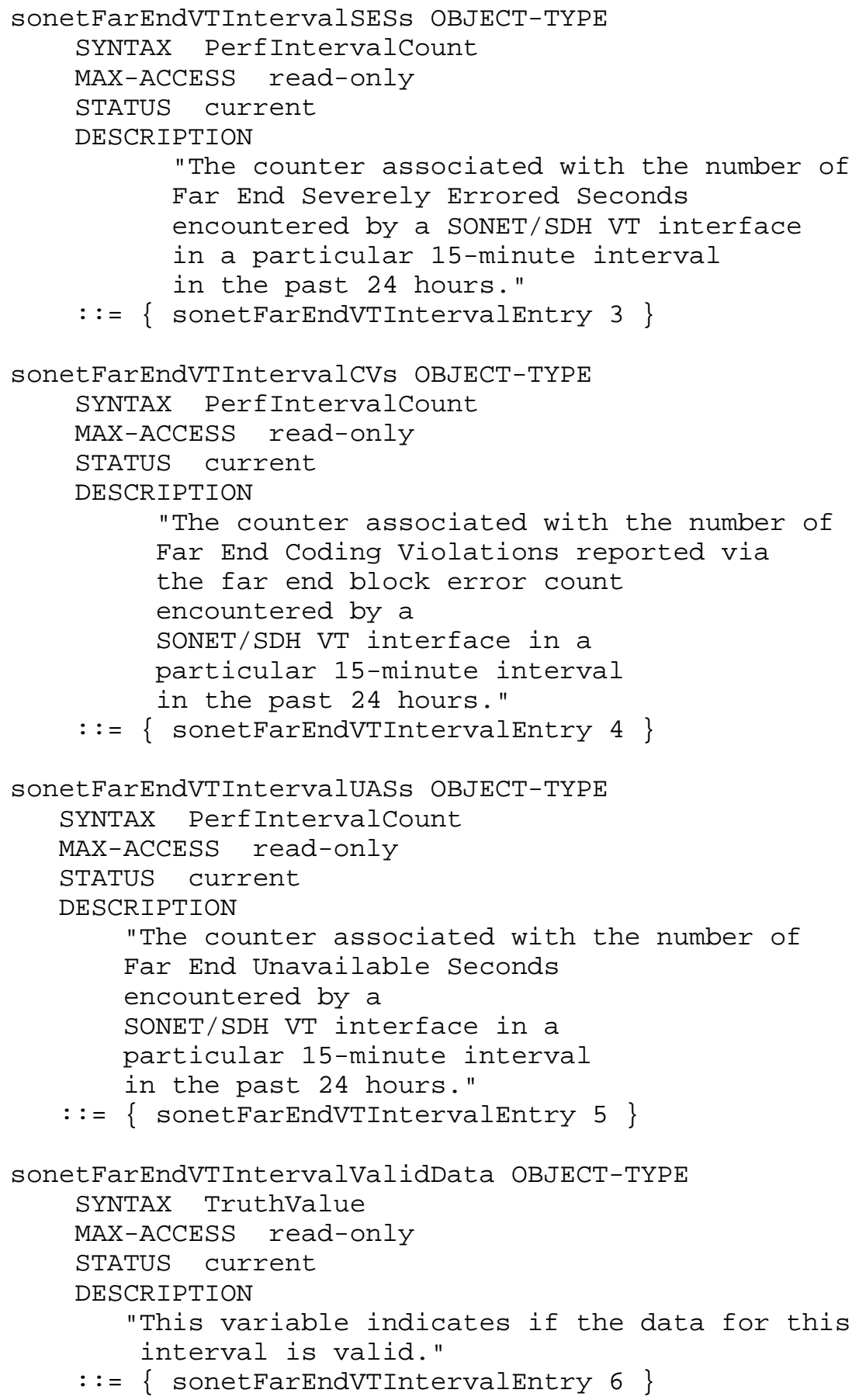


-- conformance information

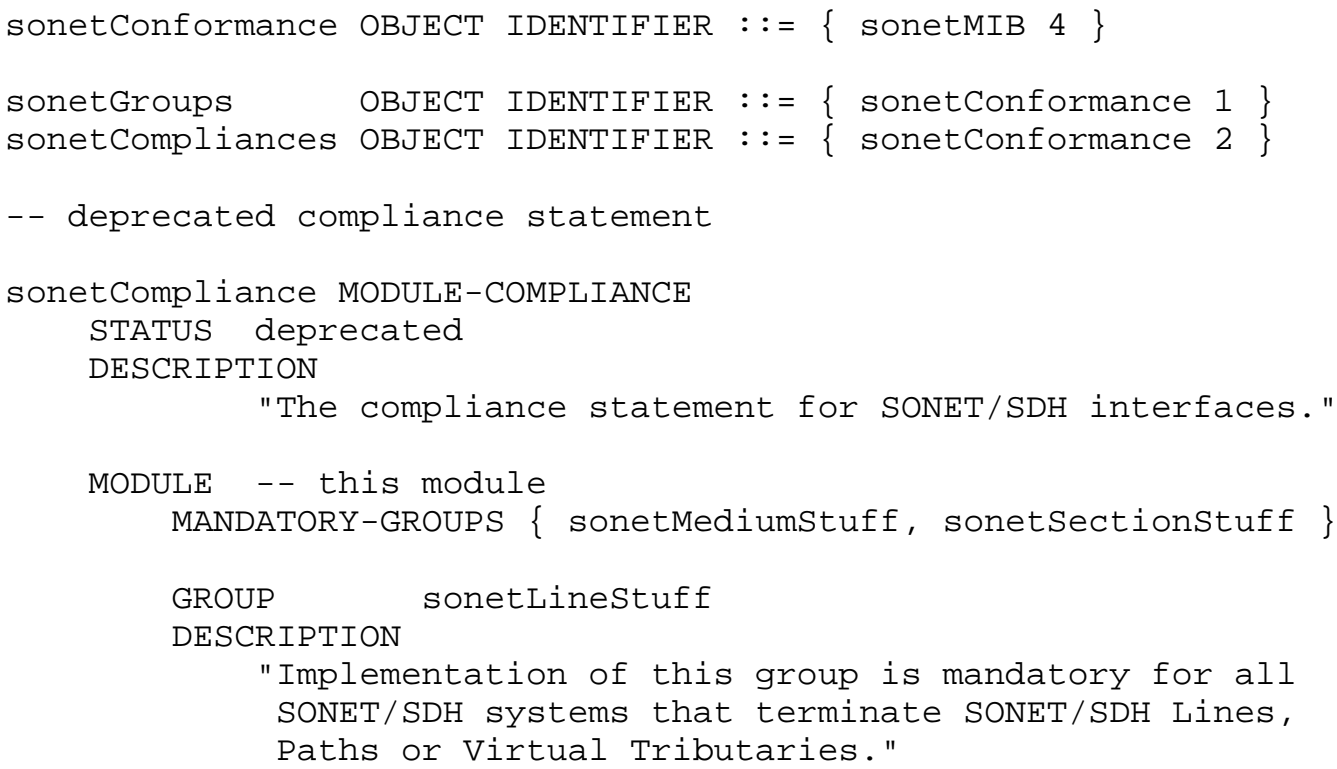




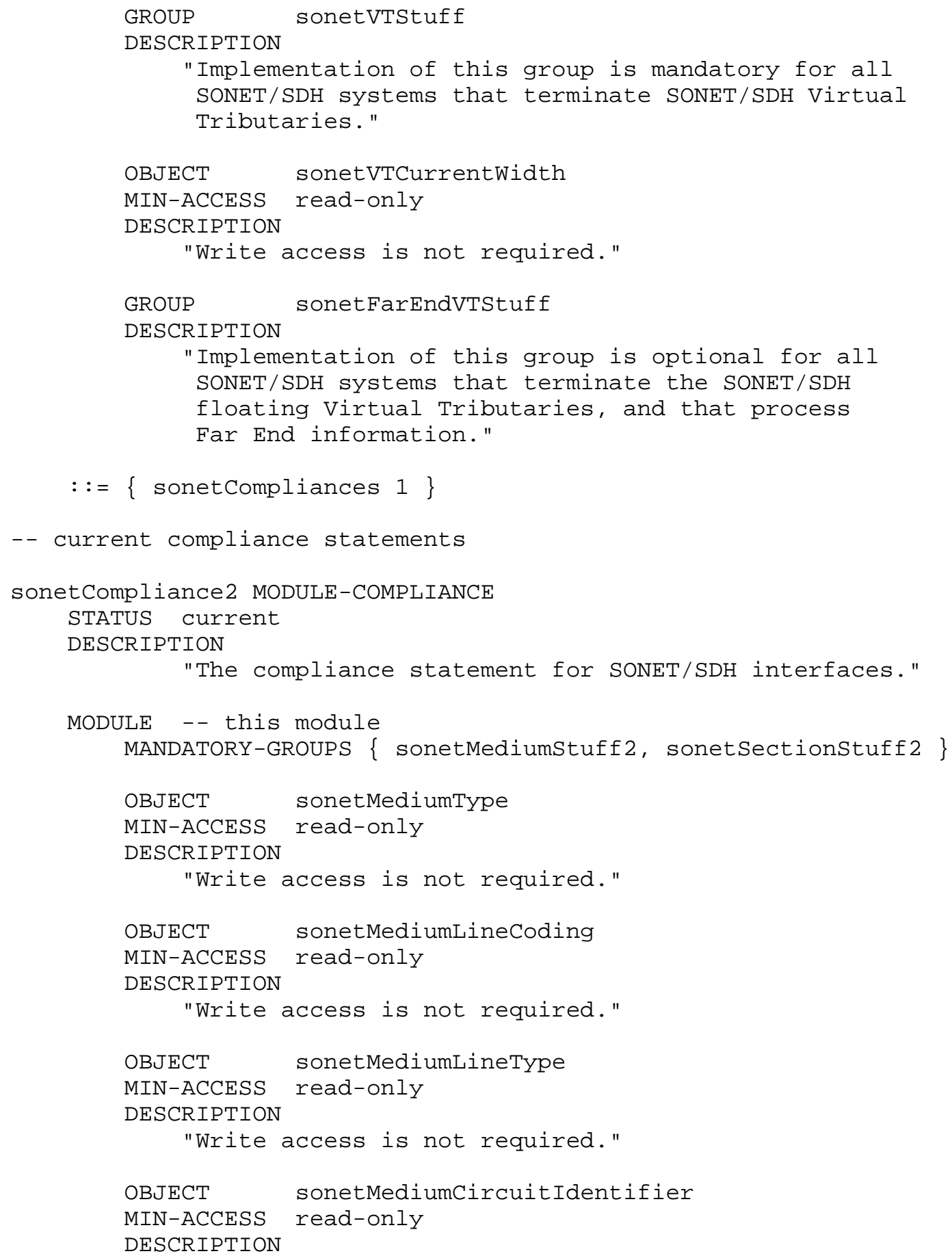




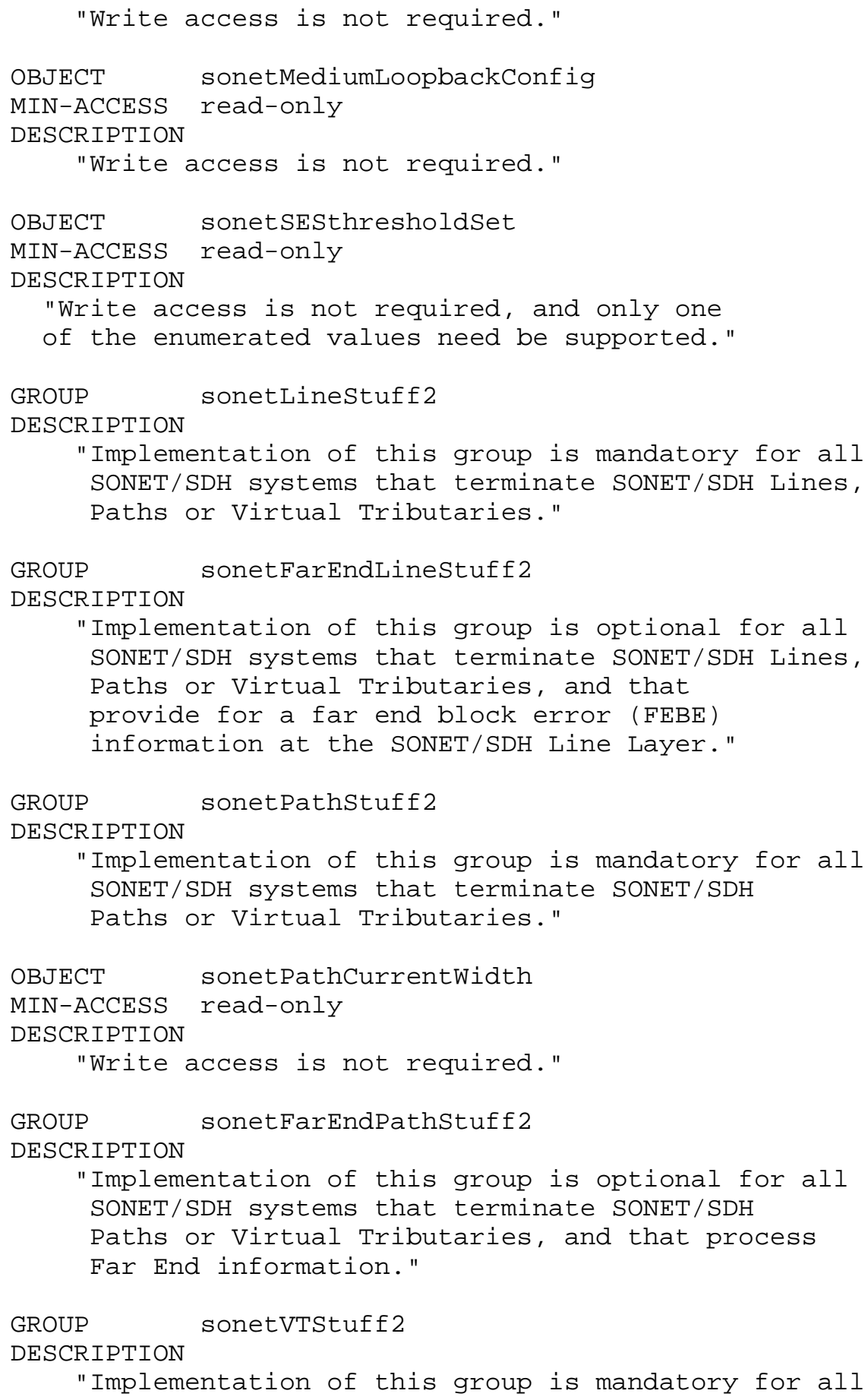




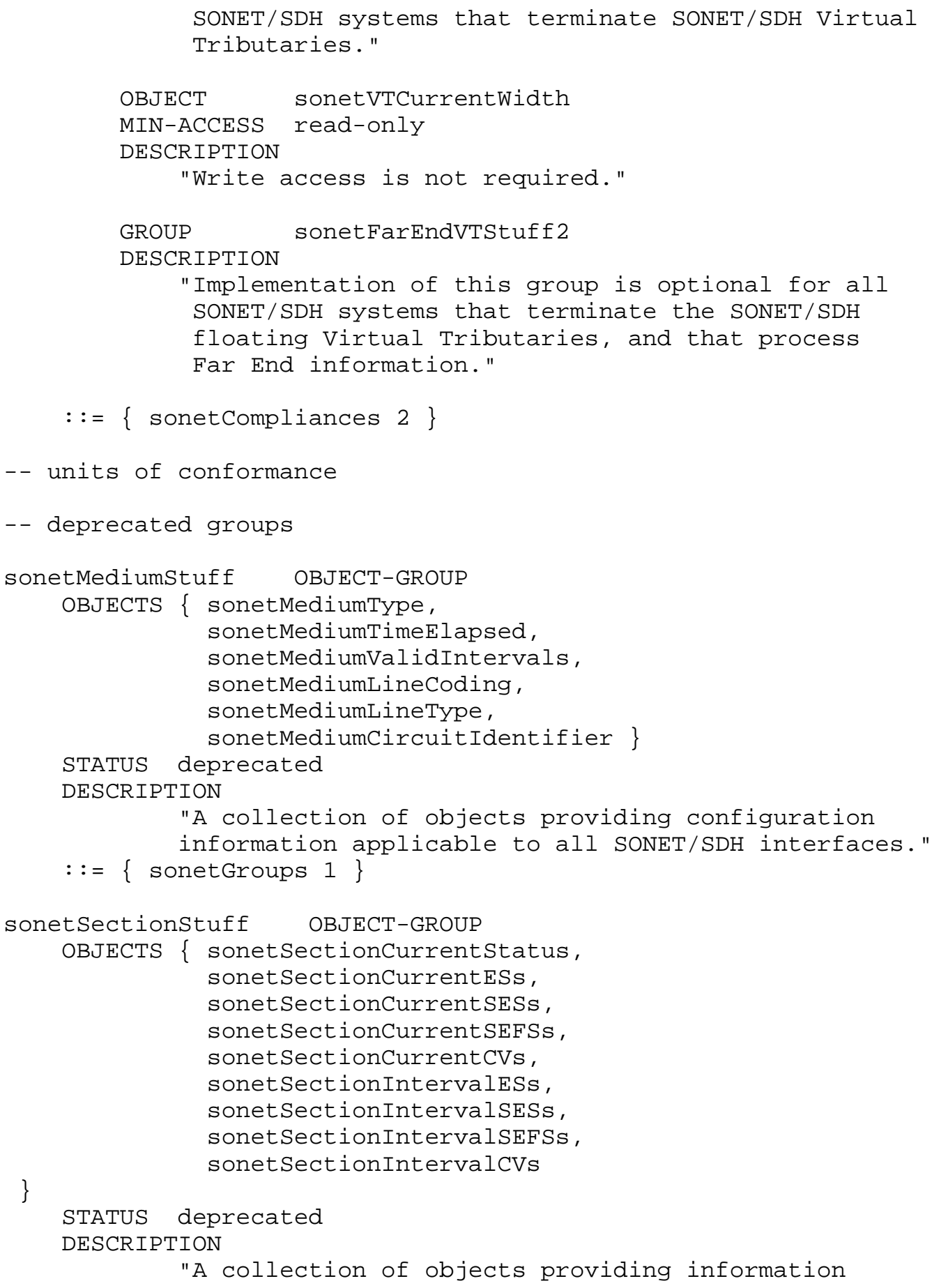

"A collection of objects providing information 


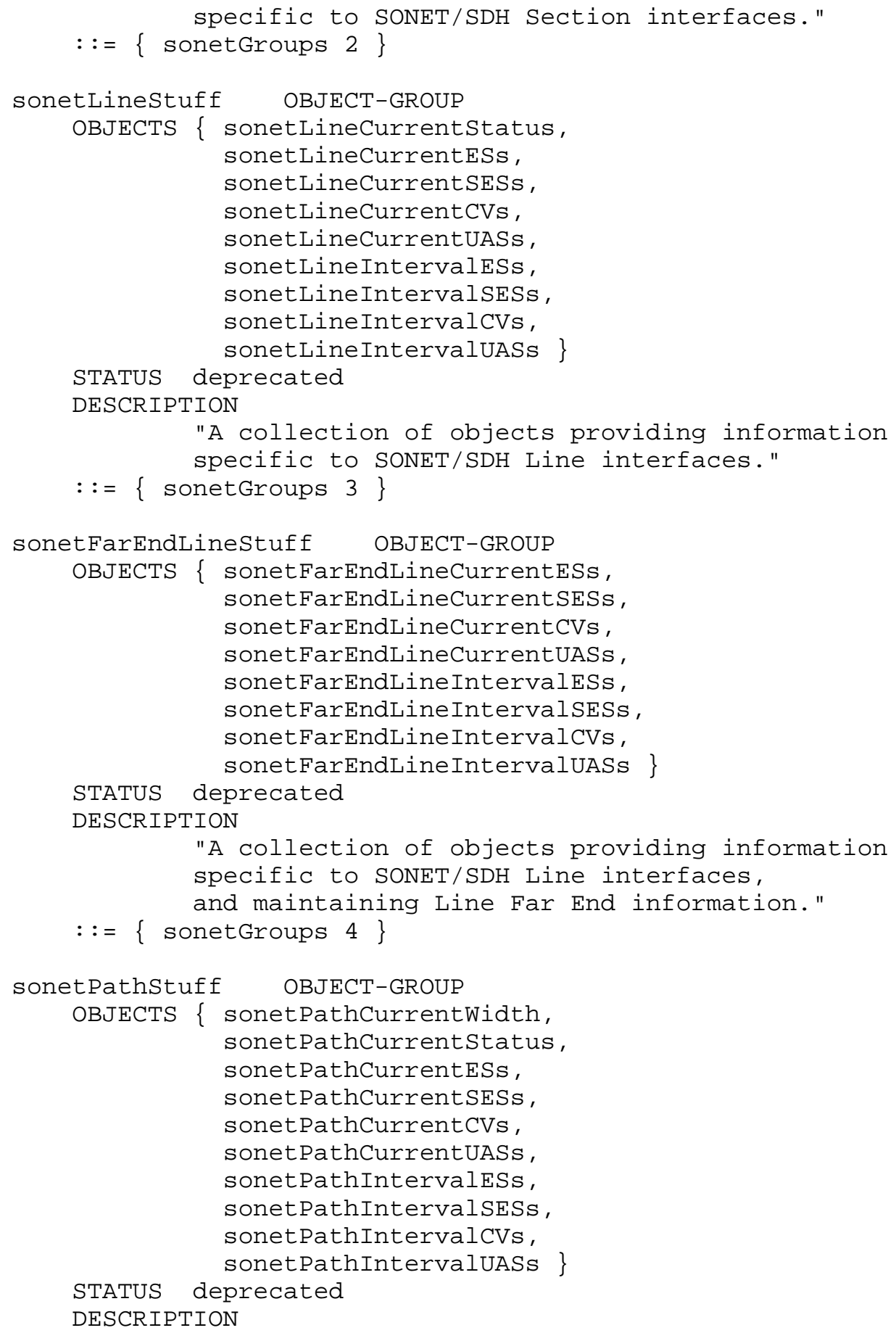


"A collection of objects providing information specific to SONET/SDH Path interfaces." $::=\{$ sonetGroups 5$\}$

sonetFarEndPathStuff OBJECT-GROUP

OBJECTS \{ sonetFarEndPathCurrentESs, sonetFarEndPathCurrentSESs, sonetFarEndPathCurrentCVs, sonetFarEndPathCurrentUASs, sonetFarEndPathIntervalESs, sonetFarEndPathIntervalSESs, sonetFarEndPathIntervalCVs, sonetFarEndPathIntervalUASs \}

STATUS deprecated DESCRIPTION

"A collection of objects providing information specific to SONET/SDH Path interfaces, and maintaining Path Far End information." $::=\{$ sonetGroups 6$\}$

sonetVTStuff OBJECT-GROUP

OBJECTS \{ sonetVTCurrentwidth, sonetVTCurrentstatus, sonetVTCurrentESs, sonetVTCurrentSESs, sonetVTCurrentCVs, sonetVTCurrentUASs, sonetVTIntervalESs, sonetVTIntervalSESs, sonetVTIntervalCVs, sonetVTIntervalUASs \}

STATUS deprecated DESCRIPTION

"A collection of objects providing information specific to SONET/SDH VT interfaces."

$::=\{$ sonetGroups 7$\}$

sonetFarEndVTStuff OBJECT-GROUP

OBJECTS \{ sonetFarEndVTCurrentESs, sonetFarEndVTCurrentSESs, sonetFarEndVTCurrentCVs, sonetFarEndVTCurrentUASs, sonetFarEndVTIntervalESs, sonetFarEndVTIntervalSESs, sonetFarEndVTIntervalCVs, sonetFarEndVTIntervalUASs \}

STATUS deprecated DESCRIPTION 


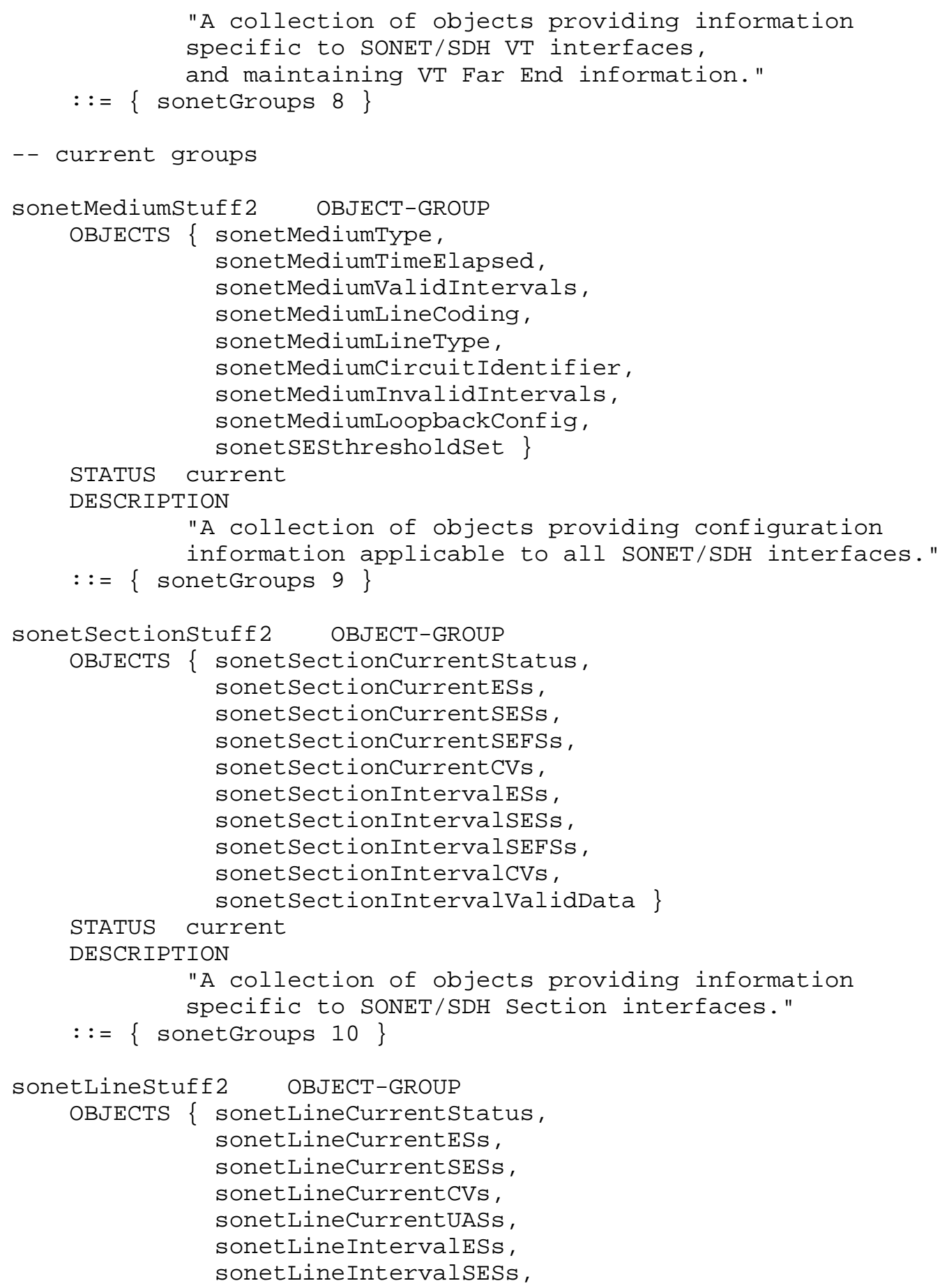

Tesink 


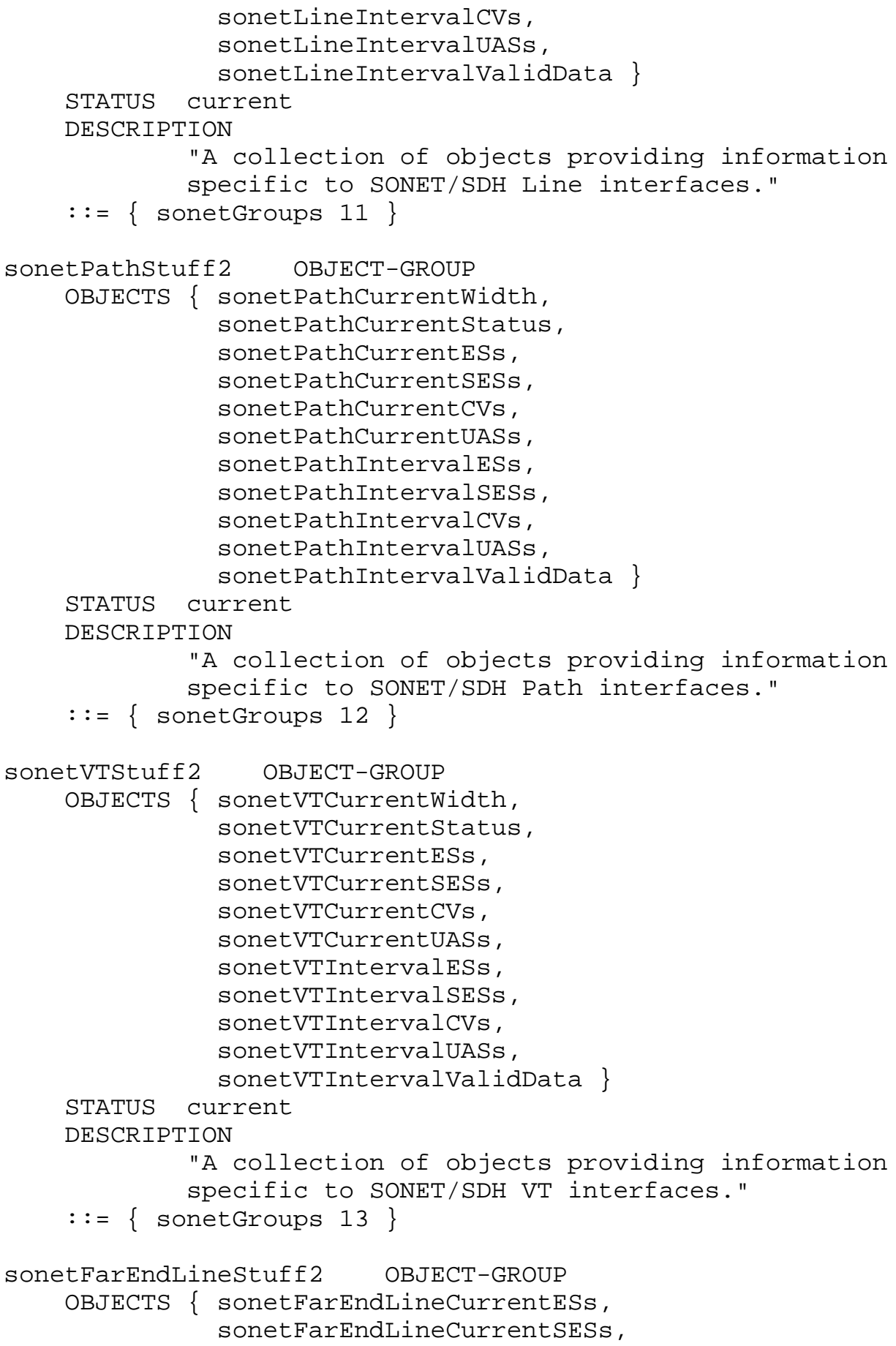




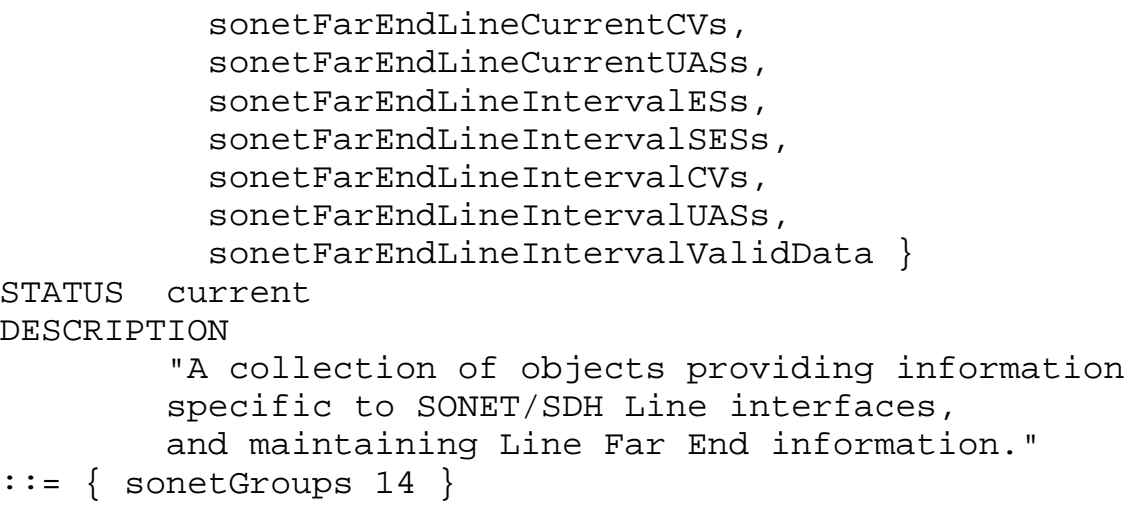

"A collection of objects providing information specific to SONET/SDH VT interfaces, and maintaining VT Far End information." $::=\{$ sonetGroups 16$\}$ 
END

5. Acknowledgments

This specification is a product of the AToM MIB Working Group. The author would like to acknowledge Mike Heard for his many valuable contributions to this memo.

6. Security Considerations

There are a number of management objects defined in this MIB module that have a MAX-ACCESS clause of read-write: sonetMediumType, sonetMediumLineCoding, sonetMediumLineType, sonetMediumCircuitIdentifier, sonetMediumLoopbackConfig, sonetSESthresholdSet, sonetPathCurrentWidth, and sonetVTCurrentWidth. It is possible for writes to these objects to have disruptive effects on network operation that range from invalid performance data to traffic interruptions. Users of this MIB module must therefore be aware that support for SET operations in a non-secure environment without proper protection can have a negative effect on network operations.

The readable objects in this MIB module (i.e., the objects with a MAX-ACCESS other than not-accessible) may be considered sensitive in some environments since, collectively, they provide extensive information about the performance of interfaces in SONET/SDH equipment or networks and can reveal some aspects of their configuration. In such environments it is important to control even GET and NOTIFY access to these objects and possibly to encrypt the values of these objects when sending them over the network via SNMP.

SNMP versions prior to SNMPv3 did not include adequate security. Even if the network itself is secure (for example by using IPSec), even then, there is no control as to who on the secure network is allowed to access and GET/SET (read/change/create/delete) the objects in this MIB module.

It is RECOMMENDED that implementers consider the security features as provided by the SNMPv3 framework (see [RFC3410], section 8), including full support for the SNMPv3 cryptographic mechanisms (for authentication and privacy).

Further, deployment of SNMP versions prior to SNMPv3 is NOT RECOMMENDED. Instead, it is RECOMMENDED to deploy SNMPv3 and to enable cryptographic security. It is then a customer/operator responsibility to ensure that the SNMP entity giving access to an 
instance of this MIB module is properly configured to give access to the objects only to those principals (users) that have legitimate rights to indeed GET or SET (change/create/delete) them.

7. References

\subsection{Normative References}

[RFC2119] Bradner, S., "Key words for use in RFCs to Indicate Requirements Levels", BCP 14, RFC 2119, March 1997.

[RFC2578] MCCloghrie, K., Perkins, D. and J. Schoenwaelder, "Structure of Management Information Version 2 (SMIv2)", STD 58, RFC 2578, April 1999.

[RFC2579] MCCloghrie, K., Perkins, D. and J. Schoenwaelder, "Textual Conventions for SMIv2", STD 58, RFC 2579, April 1999.

[RFC2580] McCloghrie, K., Perkins, D. and J. Schoenwaelder, "Conformance Statements for SMIv2", STD 58, RFC 2580, April 1999.

[RFC3593] Tesink, K., "Textual Conventions for MIB Modules Using Performance History Based on 15 Minute Intervals", RFC 3593, September 2003.

[T1.105a] American National standard for Telecommunications Digital Hierarchy - Optical Interface Rates and Formats Specification, ANSI T1.105-1988.

[T1.105b] American National Standard for Telecommunications Digital Hierarchy - Optical Interface Rates and Formats Specification, ANSI T1.105-1991.

[T1.106] American National Standard for Telecommunications Digital Hierarchy - Optical Interface Specification (Single-Mode), ANSI T1.106-1988.

[T1M1.3] Draft American National Standard for Telecommunications - Digital Hierarchy - Layer 1 In-Service Digital Transmission Performance Monitoring, T1M1.3/93-005R2, July 1993.

[G.707] CCITT Recommendation G.707, "Synchronous Digital Hierarchy Bit Rates", June 1992. 
[G.708]

[G.709]

$[G .783]$

$[\mathrm{RFC} 1213]$

[RFC2863]

[RFC2864]

$[\mathrm{T} 1.231 \mathrm{a}]$

[TR253]

[G.826]

[GR253]

$[\mathrm{T} 1.231 \mathrm{~b}]$
CCITT Recommendation G.708, "Network Node Interface for the Synchronous Digital Hierarchy", June 1992.

CCITT Recommendation G.709, "Synchronous Multiplexing Structure", June 1992.

CCITT Recommendation G.783, "Characteristics of Synchronous Digital Hierarchy (SDH) Multiplexing Equipment Functional Blocks", November 1992.

McCloghrie, K. and M. Rose, "Management Information Base for Network Management of TCP/IP-based internets: MIBII", RFC 1213, March 1991.

McCloghrie, K. and F. Kastenholz, "The Interfaces Group MIB", RFC 2863, June 2000.

McCloghrie, K. and G. Hanson, "The Inverted Stack Table Extension to the Interfaces Group MIB", RFC 2864, June 2000 .

American National Standard for Telecommunications Digital Hierarchy - Layer 1 In-Service Digital Transmission Performance Monitoring, ANSI T1.231-1993, September 1993.

Bellcore TR-NWT-000253, Issue 1, "Synchronous Optical Network (SONET) Transport Systems: Common Generic Criteria", December 1991.

ITU Recommendation G.826, "Error Performance Parameters and Objectives for International Constant Bit Rate Digital Paths at or above Primary Rate", September 1995 (COM 13-R57E).

Bellcore GR-253-CORE, Issue 2, "Synchronous Optical Network (SONET) Transport Systems Common Generic Criteria", December 1995.

American National Standard for Telecommunications Digital Hierarchy - Layer 1 In-Service Digital Transmission Performance Monitoring, ANSI T1.231-1997, September 1997. 


\title{
7.2. Informative References
}

[RFC1595] Brown, T. and K. Tesink, "Definitions of Managed Objects for the SONET/SDH Interface Type", RFC 1595, March 1994.

[RFC2495] Fowler, D., "Definitions of Managed Objects for the DS1, E1, DS2 and E2 Interface Types", RFC 2495, January 1999.

[RFC2496] Fowler, D., "Definitions of Managed Objects for the DS3/E3 Interface Type", RFC 2496, January 1999.

[RFC2558] Tesink, K., "Definitions of Managed Objects for the SONET/SDH Interface Type", RFC 2558, March 1999.

[RFC3410] Case, J., Mundy, R., Partain, D. and B. Stewart, "Introduction and Applicability Statements for InternetStandard Management Framework", RFC 3410, December 2002.

8. Intellectual Property statement

\begin{abstract}
The IETF takes no position regarding the validity or scope of any intellectual property or other rights that might be claimed to pertain to the implementation or use of the technology described in this document or the extent to which any license under such rights might or might not be available; neither does it represent that it has made any effort to identify any such rights. Information on the IETF's procedures with respect to rights in standards-track and standards-related documentation can be found in BCP-11. Copies of claims of rights made available for publication and any assurances of licenses to be made available, or the result of an attempt made to obtain a general license or permission for the use of such proprietary rights by implementors or users of this specification can be obtained from the IETF secretariat.

The IETF invites any interested party to bring to its attention any copyrights, patents or patent applications, or other proprietary rights which may cover technology that may be required to practice this standard. Please address the information to the IETF Executive Director.
\end{abstract}


Appendix A: The delay-line approach to statistics collection.

According to ANSI T1.231 unavailable time begins at the onset of 10 contiguous severely errored seconds -- that is, unavailable time starts with the first of the 10 contiguous SESs -- and while an interface is deemed unavailable all counters for that interface are frozen except for the UAS count. Since changes in the signal state lag the data to which they apply by 10 seconds, an implementation which wishes to avoid making retroactive adjustments to the counts must pass the the one-second statistics through a 10-second delay line prior to updating any counters. That can be done by performing the following steps at the end of each one second interval.

i) Read near/far end line and path CV counts and alarm status flags from the hardware.

ii) Accumulate the CV counts for the preceding second and compare them to the ES and SES threshold for the layer in question. Update the signal state and shift the one-second CV counts and ES/SES flags into the 10-element delay line. Note that far-end one-second statistics are to be flagged as "absent" during any second in which there is an incoming defect at the layer in question or at any lower layer.

iii) Update the current interval statistics using the signal state from the previous update cycle and the one-second CV counts and ES/SES flags shifted out of the 10-element delay line.

This procedure guarantees that the statistical counters will be correctly updated at all times, although they lag real time by 10 seconds. It is illustrated in the figure below. At the end of each 15 minutes interval the current interval counts are transferred to the most recent interval entry and each interval is shifted up by one position, with the oldest being discarded if necessary in order to make room. The current interval counts then start over from zero. Note, however, that the signal state calculation does not start anew at each interval boundary; rather, signal state information is retained across interval boundaries. 


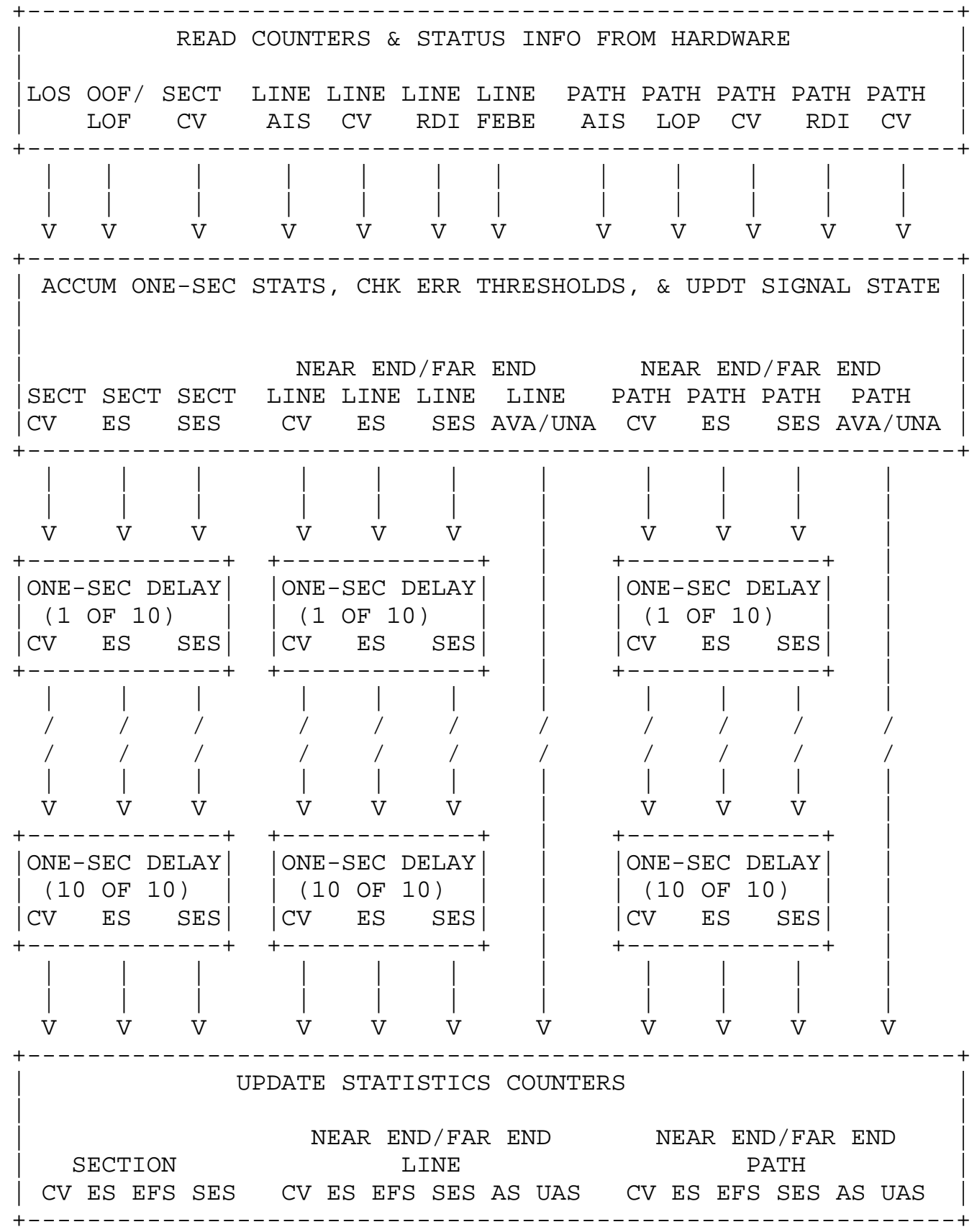

Note that if such a procedure is adopted there is no current interval data for the first ten seconds after a system comes up. 
noSuchInstance must be returned if a management station attempts to access the current interval counters during this time.

It is an implementation-specific matter whether an agent assumes that the initial state of the interface is available or unavailable.

Appendix B - RFC 1595 SES interpretation

This appendix contains the values for $x$ for the Section, Line, Path, and VT Layers as used in [T1M1.3] [RFC1595][TR253].

Value for $x$ for SONET/SDH Section SES Definition

Rate $\mathrm{x} \quad$ Minimum Bit Error Rate

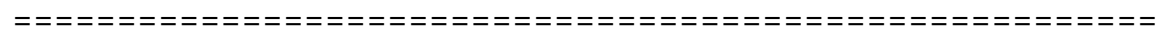

$\begin{array}{lrr}\text { OC }-1 & 9 & 1.5 \times 10^{\wedge}-7 \\ \text { OC-3 } & 16 & 1 \times 10^{\wedge}-7 \\ \text { OC-9 } & 47 & 1 \times 10^{\wedge}-7 \\ \text { OC-12 } & 63 & 1 \times 10^{\wedge}-7 \\ \text { OC-18 } & 94 & 1 \times 10^{\wedge}-7 \\ \text { OC }-24 & 125 & 1 \times 10^{\wedge}-7 \\ \text { OC }-36 & 187 & 1 \times 10^{\wedge}-7 \\ \text { OC }-48 & 249 & 1 \times 1 \times\end{array}$

Value for $x$ for SONET/SDH Line SES Definition

$\begin{array}{lcc}\text { Rate } & x & \begin{array}{c}\text { Minimum } \\ \text { Bit Error Rate }\end{array} \\ ===================================== \\ \text { OC-1 } & 12 & 2 \times 10^{\wedge}-7 \\ \text { OC-3 } & 32 & 2 \times 10^{\wedge}-7 \\ \text { OC-9 } & 47 & 2 \times 10^{\wedge}-7 \\ \text { OC-12 } & 124 & 2 \times 1 \\ \text { OC-18 } & 186 & 2 \times 10^{\wedge}-7 \\ \text { OC-24 } & 248 & 2 \times 10^{\wedge}-7 \\ \text { OC-36 } & 370 & 2 \times 10^{\wedge}-7 \\ \text { OC-48 } & 494 & 2 \times 10^{\wedge}-7 \\ & & 2 \times 10^{\wedge}-7\end{array}$

Value for $x$ for SONET/SDH STS-Path SES Definition

$\begin{array}{lcc}\text { Rate } & x & \text { Minimum Bit Error Rate } \\ ======================================= \\ \text { STS }-1 \\ \text { STS }-3 & 9 & 1.5 \times 10^{\wedge}-7 \\ 16 & 1 \times 10^{\wedge}-7\end{array}$


Value for $x$ for SONET/SDH VT-Path SES Definition

\begin{tabular}{|c|c|c|c|}
\hline \multirow{2}{*}{$\begin{array}{l}\text { Rate } \\
====\end{array}$} & $\mathrm{x}$ & Minimum Bit & Error Rate \\
\hline & $===$ & $===========$ & $===========$ \\
\hline VT1. 5 & 4 & 2 & $\times 10^{\wedge}-6$ \\
\hline VT2 & 6 & 2 & $x 10^{\wedge}-6$ \\
\hline VT3 & 8 & 2 & $\times 10^{\wedge}-6$ \\
\hline VT 6 & 14 & 2 & $\times 10^{\wedge}-6$ \\
\hline
\end{tabular}

Author's Address

Kaj Tesink

Telcordia Technologies

331 Newman Springs Road

P.O. Box 7020

Red Bank, NJ 07701-7020

Phone: (732) 758-5254

EMail: kaj@research.telcordia.com 
Full Copyright statement

Copyright (C) The Internet Society (2003). All Rights Reserved.

This document and translations of it may be copied and furnished to others, and derivative works that comment on or otherwise explain it or assist in its implementation may be prepared, copied, published and distributed, in whole or in part, without restriction of any kind, provided that the above copyright notice and this paragraph are included on all such copies and derivative works. However, this document itself may not be modified in any way, such as by removing the copyright notice or references to the Internet society or other Internet organizations, except as needed for the purpose of developing Internet standards in which case the procedures for copyrights defined in the Internet Standards process must be followed, or as required to translate it into languages other than English.

The limited permissions granted above are perpetual and will not be revoked by the Internet society or its successors or assigns.

This document and the information contained herein is provided on an "AS IS" basis and THE INTERNET SOCIETY AND THE INTERNET ENGINEERING TASK FORCE DISCLAIMS ALL WARRANTIES, EXPRESS OR IMPLIED, INCLUDING BUT NOT LIMITED TO ANY WARRANTY THAT THE USE OF THE INFORMATION HEREIN WILL NOT INFRINGE ANY RIGHTS OR ANY IMPLIED WARRANTIES OF MERCHANTABILITY OR FITNESS FOR A PARTICULAR PURPOSE.

Acknowledgement

Funding for the RFC Editor function is currently provided by the Internet society. 